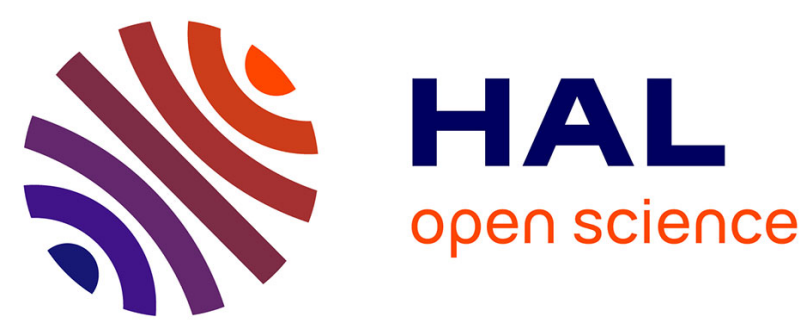

\title{
Performance Control for Interconnection of Identical Systems: Application to PLL network design
}

\author{
Anton Korniienko, Gérard Scorletti, Eric Colinet, Eric Blanco
}

\section{To cite this version:}

Anton Korniienko, Gérard Scorletti, Eric Colinet, Eric Blanco. Performance Control for Interconnection of Identical Systems: Application to PLL network design. International Journal of Robust and Nonlinear Control, 2016, 26 (1), pp.3 - 27. 10.1002/rnc.3285 . hal-01083781

\section{HAL Id: hal-01083781 https://hal.science/hal-01083781}

Submitted on 18 Nov 2014

HAL is a multi-disciplinary open access archive for the deposit and dissemination of scientific research documents, whether they are published or not. The documents may come from teaching and research institutions in France or abroad, or from public or private research centers.
L'archive ouverte pluridisciplinaire HAL, est destinée au dépôt et à la diffusion de documents scientifiques de niveau recherche, publiés ou non, émanant des établissements d'enseignement et de recherche français ou étrangers, des laboratoires publics ou privés. 


\title{
Performance Control for Interconnection of
}

Identical Systems: Application to PLL

\author{
network design
}

Article accepted for the publication in International

Journal of Nonlinear and Robust Control

\author{
A. Korniienko ${ }^{\ddagger} \triangle$, G. Scorletti ${ }^{\triangleleft}$ Eric Colinet $^{\ddagger}$ Eric Blanco $^{\triangle}$ \\ ‡ CEA, LETI, MINATEC, 17 rue des martyrs, 38054 Grenoble Cedex 9, France. \\ $\triangle$ Laboratoire Ampère, Ecole Centrale de Lyon, 69134 Ecully Cedex, France. \\ anton.korniienko@ec-lyon.fr
}

November 18, 2014

\begin{abstract}
In this paper, the problem of the control law design for interconnected identical
\end{abstract}


systems ensuring the global stability and the global performance properties is under consideration. Inspired by the decentralized control law design methodology using the dissipativity input-output approach, the problem is reduced to the problem of satisfying two conditions: (i) the condition on the interconnection and (ii) the condition on the local subsystem dynamics. Both problems are efficiently solved applying a (quasi-) convex LMI optimization and standard $H_{\infty}$ synthesis. The proposed design methodology is applied to the control law design of a synchronous PLL network.

Keywords Multi-Agent systems, decentralized control, $H_{\infty}$ control, dissipativity input-output approach, LMI optimization, PLL network synchronization.

\section{Introduction}

The design problem of a large-scale system represented as an interconnection of identical subsystems is under consideration. Each subsystem is a feedback interconnection of the plant and the controller. The problem of system design is: given the plant and the interconnection topology, find the subsystem controllers ensuring an appropriate global behavior of the overall system, referred to as the global system performance.

This design problem turns out to be relevant in various fields of science and engineer applications [1]. In this paper, as an example of application, we investigate the control law design for an active clock distribution network, that is, interconnected Phase-Locked Loops (PLLs) interconnected in an array. This device was proposed as an alternative to the classical clock distribution trees in order to distribute the clock signal in a synchronous multi-core microprocessor system with a better level of performance in terms of perturbation 
rejection, robustness and power consumption [2-5]. The active clock distribution network design consists in computing the controller (filter) of the (feedback) PLL loop in order to ensure a high level of global system performance. Indeed, the global synchronization of the phase and frequency in a specified amount of time is crucial to ensure the correct operation of the clock network. Issues of minimal global system bandwidth, maximal control signal level, noise and perturbation rejection must be addressed as well. The two first requirements enforce the convergence speed and limit the power consumption of the whole system, while the two last ones are critical to distribute the clock properly in a noisy and often perturbed chip environment.

In the Microelectronics field, the traditional design procedure of the PLL network system usually consists of two steps: (i) local design of the PLL (filter) and (ii) global analysis of the PLL network. During the first step, the network interconnection is neglected, that is, the subsystems (PLLs) are assumed independent. The local subsystem design, usually based on the classical frequency design methods, is then performed such that the local subsystem behavior or local subsystem performance are satisfactory. The second step consists in the performance analysis of the global system composed of the designed subsystems interconnected with the given topology. Usually, to perform this step, some local and global simulations are performed in order to test if the local performance ensured by the local design is achieved for the overall system as well. If it is not the case, the difference between the local performance and global one is then estimated i.e. the global performance is rather evaluated in the relative than in the absolute way. This design procedure is a reminiscence of some iterative, "trial and error" approaches to decentralized control [6]. 
The same design procedure, i.e. the local subsystem design followed by the global system analysis, can be applied to the design of other large-scale interconnected systems. However, due to the mutual coupling, the multiple interconnections and feedbacks inside the network, the traditional design method can fail. In general, it cannot ensure that the local performance of the independent subsystem will not be significantly degraded when the subsystem is connected to the global network. Moreover, even the global system stability is not ensured. Hence, the impact of the global network interconnections can be very important: it is then necessary to design the control law under the constraint of global stability and global performance.

Though the (heuristic) traditional design method does not ensure a priori appropriate global system behavior, there are some motivations for its use. A first motivation is the inherent complexity of the networked system design, due to the decentralization and the possibly important dimension. Since the network topology does not connect all the subsystems with all the other ones and since for practical realization reasons, the controllers are decentralized, the design problem is a particular case of the decentralized control design problem. The structural (decentralized) constraint implies the complexity of the design problem and the inefficiency of its resolution algorithms [7]. Second, since the overall system can have a large number of subsystems, its direct global design using optimization can lead to a large number of decision variables. In the traditional approach, the design is first performed for a single local subsystem, that is an optimization problem with a significantly reduced number of decision variables. Moreover, since in the considered application the subsystems are identical, they are all designed at once. Another motivation is the existence of the standard 
and well-known local subsystem design tools, which is also the case for fields different from Microelectronics.

The main goal of this paper is to propose a new design method which is close to the Microelectronics traditional design procedure but solves its major drawbacks i.e. the local design of the subsystem does not guarantee the stability and the performance of the network. A strong motivation of procedure close to the traditional one is to improve the acceptability of the proposed design method. Furthermore, it has to be general enough to be applied to other similar design problems of networked systems, in other application fields.

Let us first discuss the existing interconnected system design methods in Control Theory with respect to our goals. A similar control problem was largely investigated in the field of Multi-Agent system. A Multi-Agent system is a network of intelligent subsystems called agents, where each agent exchanges some information with its neighbour ones, in order to achieve some specified global network behaviour. The agent is the feedback of a to-becontrolled plant and a controller.

The most fundamental result in the case of Linear Time Invariant (LTI) identical MultiAgent system is the necessary and sufficient stability conditions presented in [8]. By some aspects, this result is a generalization of the Nyquist stability criterion to Multi-Agent systems. Based on the Graph Theory, the authors transform the global stability condition of the $N$ interconnected identical Multi-Agents into local conditions of simultaneous stability for the $N$ independent different subsystems, see Theorem 3 in [8].

Based on this result, a large number of papers were published during the last years for sometimes particular classes of interconnections (Laplacian, Adjacency, symmetric or 
normal matrices). The static feedback control of [9] or the dynamic observer based solution presented in [10-12] and [13] are such examples. However, these results cannot be applied to our application of PLL network design. First, for the control law discussed in [10-13] a communication network which is able to transmit arbitrary information is assumed: plant state vector for the static control case and observer based controller state vectors for the dynamic control case. Furthermore, the computation of these controllers and observers is not investigated. The reason is the intrinsic complexity of the simultaneous stabilization problem for three and more subsystems, as discussed in [14]. This fact is confirmed by the results presented in $[15,16]$ where the control law computation is based on Bilinear Matrix Inequality (BMI) optimization which, in general, cannot be efficiently solved.

Another drawback of these results which is important for our application, is that the proposed control law design methods do not address general performance specifications. The focus is only on the consensus or coordination with a fixed convergence rate, inspired by Theorem 3 in [8]. For example, the specification of the reference ramp tracking, necessary for the PLL synchronization, cannot be considered. The minimization of the $H_{\infty}$ norm of a global transfer function in [17] is an interesting perspective for considering some performance specifications. However, the solution presented in [17] could be extremely conservative since it is based on a quadratic Lyapunov function which is common to all the subsystems with a block diagonal structure. A less conservative result is presented in [18], where, if the interconnection satisfies a structural property, the global system could be equivalently decomposed into independent subsystems. In this case, the design of controller ensuring the global stability and an $H_{\infty}$ norm inequality on a global transfer function is investigated. 
This inequality ensures that the magnitude of the frequency response of the global transfer function is below a frequency independent constraint. As in most of the applications, the performance specifications of the PLL network have to be expressed as "the magnitude of the frequency response of the global transfer function is below a frequency dependent constraint". The introduction of performance inputs and outputs to the global system associated to weighting functions [19] in order to deal with frequency dependent constraint could destroy the system structural property so that the global system might not be decomposed. In this case the approaches presented in $[17,18]$ cannot be directly applied.

The second possible approach for the large-scale system design is the Decentralized Control design methods, see [20] and [21]. In these approaches, a system is represented as the interconnection of subsystems. A local controller is designed for each subsystem such that the global and sometimes local system objectives are ensured. These (local) controllers form hence the decentralized controller.

Despite a large amount of works, the design of such controllers is still an open problem for the general case [22-24]. Under some assumptions, it is proved to be NP-hard [7] and boils down to a non convex BMI optimization problem [24,25]. A first approach to solve the problem is to propose a direct but heuristic resolution of the BMI problem [24] or to over constraint the problem to obtain a convex formulation. The last approach is adopted in the paper [22] where a quadratic Lyapunov function is fixed to have a block diagonal matrix structure. A second approach consists in a relaxation of the complex structural constraints imposed by the network topology in order to obtain an efficient design algorithm as in [26]. The authors of [26] propose to solve the problem introducing the link between global system 
and local subsystem behaviours based on a subsystem dissipativity characterisation used in the input-output approaches [27].

Nevertheless, the approach presented in [26] is not suitable for our application. In [26], the subsystems are not necessarily identical and thus the complexity of the design conditions strongly depends on the number of subsystems. This potentially leads to large optimization problems since, for every single subsystem, a set of Linear Matrix Inequality (LMI) constraints has to be introduced. The application to the PLL network design can be conservative since this methods does not consider identical subsystems, in contrast with the Multi-Agent approaches as in [8]. Furthermore, the proposed approach does not satisfy our goal, that is, propose a procedure where the design of a local controller implies the stability and the performance of the global system.

In this paper, we propose a design method for the control of interconnections of identical subsystems. The proposed approach is to design the (local) controller of a subsystem in order to achieve the stability and the specifications of performance for the interconnections of identical subsystems (the global system). A first result reveals how to compute the local controller such that the global system is stable, with the $H_{\infty}$ norm of a global transfer function less than a given level $\eta$ (section 2). The input-output behavior of the subsystem is described by a general dissipative property [27], that is, the input signals and the output signals satisfy an (integral) quadratic constraint. Such an approach is suitable to obtain stability criteria for interconnected systems, using separation of graph arguments [27-30]. The nice feature is that the local controller can be designed by the standard $H_{\infty}$ method with some additional LMI constraints due to the interconnection. Based on this first result, 
a second one reveals how to obtain a similar result with the $H_{\infty}$ norm inequality replaced by a frequency dependent constraint on the frequency response magnitude of the global transfer function (section 3). The nice feature is that this kind of constraints allows to address typical design specifications. The proposed method is then applied to the design of a PLL network (section 4). The nice feature is that we succeed in improving the traditional design process by a priori ensuring the global stability and performance.

Notations The superscript " $T$ " defines a real matrix transpose while the superscript "*" defines the complex conjugate transpose. $I_{N}$ denotes a square $N \times N$ identity matrix while $0_{n \times m}$ is a $n \times m$ zero matrix. The dimension of the identity or zero matrix is omitted ( $I$ and 0 ) if it is clear from the context. The diagonal aggregation of two matrices $A$ and $B$ is denoted by $\operatorname{diag}(A, B)$. The Kronecker product between two matrices $A$ and $B$ denoted by $\otimes$ is defined as:

$$
A \otimes B=\left[a_{i j} B\right]
$$

With $G=\left[\begin{array}{ll}G_{11} & G_{12} \\ G_{21} & G_{22}\end{array}\right], G \star K$ denotes $G \star K=G_{11}+G_{12} K\left(I-G_{22} K\right)^{-1} G_{21}$ with $\star$ the Redheffer (star) product. Similarly, $K \star G$ denotes $K \star G=G_{22}+G_{21} K\left(I-G_{11} K\right)^{-1} G_{12}$. For a stable LTI system $G,\|G\|_{\infty}$ denotes the $H_{\infty}$ norm of $G$. For a matrix $P, \bar{\sigma}(P)$ denotes the maximal singular value of the matrix $P . \mathcal{L}_{2}$ is the space of $\mathbb{R}^{n}$ square integrable valued functions defined on $\mathbb{R}$, where the norm is defined by $\|f\|_{2}=\left(\int\|f(t)\|^{2} d t\right)^{1 / 2}$. 


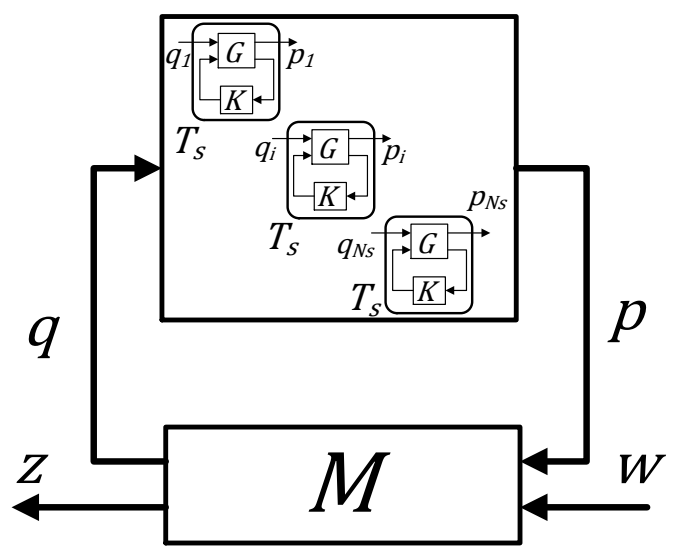

Figure 1: Considered global LTI large-scale system

\section{Large-scale control}

\subsection{Problem formulation}

The Linear Time Invariant (LTI) large-scale system with identical subsystems is defined as the interconnection $M$ of $N_{s}$ identical subsystems $T_{s}$ with $n_{s}$ inputs and $m_{s}$ outputs, that is :

$$
\begin{gathered}
p=\left(I_{N_{s}} \otimes T_{s}\right)(q) \\
{\left[\begin{array}{l}
q \\
z
\end{array}\right]=M\left(\left[\begin{array}{l}
p \\
w
\end{array}\right]\right)}
\end{gathered}
$$

where

$$
\text { - } M=\left[\begin{array}{cc}
M_{11} & M_{12} \\
M_{21} & M_{22}
\end{array}\right] \text { is a (finite dimensional) stable LTI system; }
$$

- $T_{s}=G \star K$ where the (finite dimensional) LTI systems $G$ and $K$ are referred to as the local plant and the local controller; 
- $w(t) \in \mathbb{R}^{n_{w}}$ is the system input, $z(t) \in \mathbb{R}^{n_{z}}$ the system output and $q(t) \in \mathbb{R}^{n_{s} N_{s}}$, $p(t) \in \mathbb{R}^{m_{s} N_{s}}$ are internal signals.

In the sequel, this system, denoted $\left(I_{N_{s}} \otimes T_{s}\right) \star M$ and represented Fig. 1, is referred to as the global system and $T_{s}=G \star K$ as the local system. Both systems are assumed to be well posed and causal.

Note that an active clock distribution network can be represented as a Linear Time Invariant (LTI) large-scale system with identical subsystems where the local subsystem $T_{s}$ represents a Phase-Locked Loops, $K$ the PLL filter and $M$ the interconnection of the PLLs. Remark 1. The proposed general large-scale system model (1) can describe the network of the interconnected Multi-Agents. In this particular case, the agent dynamic is represented by $T_{s}$ and their interconnection by a Laplacian (or an Adjacancy) matrix $M$ (see $[8,31]$ ). In contrast with, in the sequel, the proposed approach does not assume any particular structure of the interconnection.

Problem 1 (Decentralized control problem). Given $\eta>0$, the interconnection $M$ and the local plant $G$, find the local controller $K$ such that the global system $\left(I_{N_{s}} \otimes T_{s}\right) \star M$ is stable and

$$
\left\|\left(I_{N_{s}} \otimes T_{s}\right) \star M\right\|_{\infty}<\eta
$$

Remark 2. The proposed decentralized control problem recovers well-known Multi-Agent problems such as consensus, synchronization, coordination, reference tracking, etc. $[8,9$, $11,12,31,32]$. In this case, the minimization of the $H_{\infty}$-norm $(2)$ can be interpreted as the global agent network performance goal: minimization of the synchronisation, tracking 
or coordination error $[17,33]$. The example of the Multi-Agent synchronization will be illustrated in Section 4; the other problems can be addressed in a similar way.

\subsection{Proposed solution to the decentralized control problem}

The proposed solution is based on the description of systems using a concept of dissipativity.

Definition 1 (Dissipativity). A causal operator $H$ with the input $q$ and the output $p$ is strictly $\{X, Y, Z\}$-dissipative, where $X=X^{T}, Y$ and $Z=Z^{T}$ are real matrices with

$$
\begin{aligned}
& {\left[\begin{array}{cc}
X & Y \\
Y^{T} & Z
\end{array}\right] \text { full rank if there exists a real } \varepsilon>0 \text { such that for any } q \in \mathcal{L}_{2}} \\
& \qquad \int_{0}^{\tau}\left[\begin{array}{c}
q(t) \\
p(t)
\end{array}\right]^{T}\left[\begin{array}{cc}
X & Y \\
Y^{T} & Z
\end{array}\right]\left[\begin{array}{c}
q(t) \\
p(t)
\end{array}\right] d t \leq-\varepsilon \int_{0}^{\tau} q(t)^{T} q(t) d t
\end{aligned}
$$

if the inequalities (3) is satisfied with $\varepsilon=0$ the operator is said to be $\{X, Y, Z\}-$ dissipative.

If the operator $H$ is stable and LTI then equation (3) leads to the following frequency domain condition [34]: for almost $\forall \omega \in \mathbb{R}^{+}$and a real $\varepsilon>0$

$$
\left[\begin{array}{c}
I \\
H(j \omega)
\end{array}\right]^{*}\left[\begin{array}{cc}
X & Y \\
Y^{T} & Z
\end{array}\right]\left[\begin{array}{c}
I \\
H(j \omega)
\end{array}\right] \leq-\varepsilon I .
$$

The next theorem presents a sufficient condition for (2) based on the dissipativity properties of the interconnection $M$ and the local system $T_{s}$.

Theorem 1 (Global Performance). Given $\eta>0$, a stable interconnection $M$ and a local plant $G$ and the real matrices $X=X^{T} \in \mathbb{R}^{m_{s} \times m_{s}} \geq 0, Y \in \mathbb{R}^{m_{s} \times n_{s}}, Z=Z^{T} \in \mathbb{R}^{n_{s} \times n_{s}}$, if there exists 
(i) a positive definite matrix $P \in \mathbb{R}^{N_{s} \times N_{s}}$ such that $M$ is $\left\{\operatorname{diag}\left(P \otimes X,-\eta^{2} I\right)\right.$, $\operatorname{diag}(P \otimes Y, 0)$, $\operatorname{diag}(P \otimes Z, I)\}-$ dissipative;

(ii) a local controller $K$ such that $T_{s}=G \star K$ is strictly $\left\{-Z,-Y^{T},-X\right\}$ dissipative then the local controller $K$ ensures that the large scale system $\left(I_{N_{s}} \otimes T_{s}\right) \star M$ is stable and

$$
\left\|\left(I_{N_{s}} \otimes T_{s}\right) \star M\right\|_{\infty} \leq \eta .
$$

Discussion Condition (i) and condition (ii) of Theorem 1 can be tested using convex optimization involving Linear Matrix Inequality constraints. To this purpose, let us introduce the matrices $A, B, C, D$ which define a minimal state-space representation of $M(s)=C(s I-A)^{-1} B+D$ with $A \in \mathbb{R}^{n \times n}$. The matrix $P$ is such that the condition holds if and only if there exists a positive definite matrix $\widehat{P} \in \mathbb{R}^{n \times n}$ such that

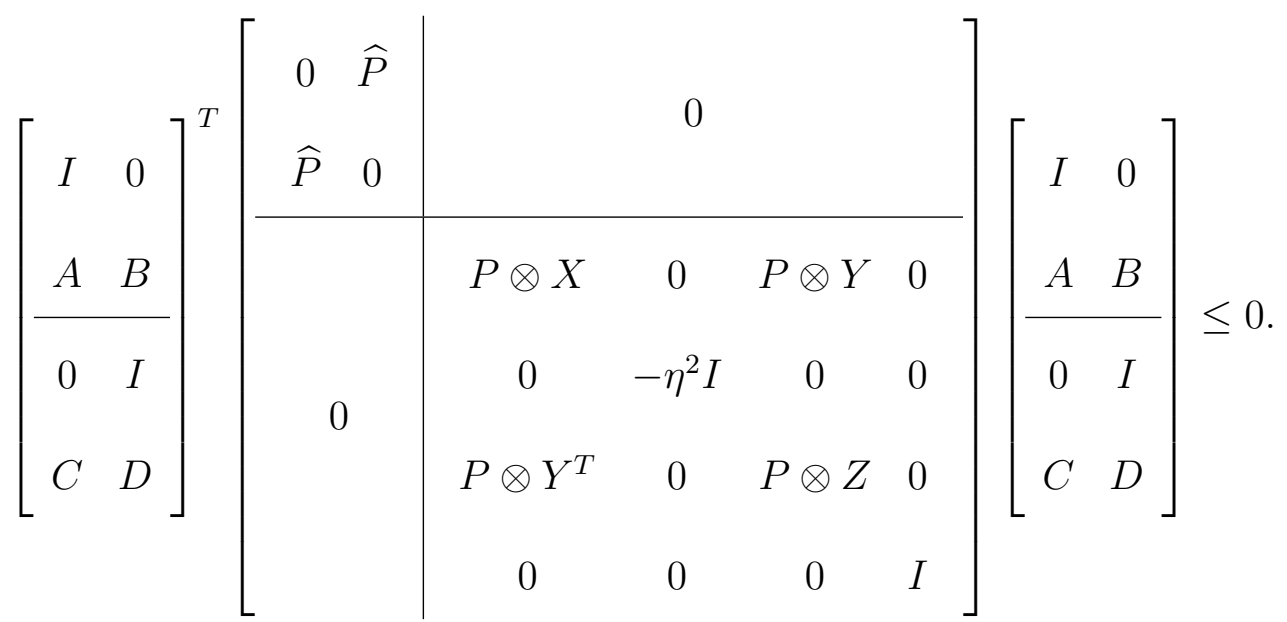

which is a Linear Matrix Inequality constraint. This inequality is directly obtained by applying the Kalman-Yakubovich-Popov (KYP) lemma [35]. For the sake of clearness and without loss of generality, in the sequel, the interconnection $M$ is considered static, that is a gain matrix. Thanks to (6), the presented results can be straightforwardly extended to the case of dynamical $M$. 
Furthermore, previously published results reveal that find a controller $K$ such that condition (ii) of Theorem 1 is satisfied can be tested using convex optimization involving Linear Matrix Inequality constraints (see e.g. [36] or [26]). Moreover, when $X>0$, using the following loop shifting transformation:

$$
\hat{T}=(-X)^{\frac{1}{2}}\left(T_{s}+X^{-1} Y\right)\left(Z-Y^{T} X^{-1} Y\right)^{-\frac{1}{2}}
$$

the computation of the controller $K$ such that $T_{s}=G \star K$ is strictly $\left\{-Z,-Y^{T},-X\right\}-$ dissipative is equivalent to the computation of $K$ such that the $\|\hat{T}\|_{\infty}<1$ which is a standard $H_{\infty}$ control problem [19]. Such a loop shifting transformation is well-posed since, by applying the Schur Lemma [37], $X>0$ and (4) imply that $Z-Y^{T} X^{-1} Y>0$.

Theorem 1 is now specialized to the stability problem.

Corollary 1 (Global Stability). Given $\eta>0$, a stable interconnection $M$, a plant $G$, and real matrices $X=X^{T} \in \mathbb{R}^{m_{s} \times m_{s}} \geq 0, Y \in \mathbb{R}^{m_{s} \times n_{s}}, Z=Z^{T} \in \mathbb{R}^{n_{s} \times n_{s}}$, if there exists

(i) a positive definite matrix $P \in \mathbb{R}^{N_{s} \times N_{s}}$ such that $M_{11}$ is $\{P \otimes X, P \otimes Y, P \otimes Z\}$-dissipative;

(ii) a local controller $K$ such that $T_{s}=G \star K$ is strictly $\left\{-Z,-Y^{T},-X\right\}$-dissipative then the local controller $K$ ensures that the large scale system $\left(I_{N_{s}} \otimes T_{s}\right) \star M$ is stable.

Remark 3. For different choices of the matrices $X, Y$ and $Z$, Corollary 1 recovers some existing results. Note that $\{0, I, 0\}$-dissipativity corresponds to passivity, while $\{I, 0, I\}$-dissipativity corresponds to (small) $\mathcal{L}_{2}$-gain. In this sense and when the non-linear counterparts of $T_{s}$ and $M_{11}$ are considered, Corollary 1 is an generalization of respectively the passivity theorem 
and the small gain one. The theorems were applied for ensuring the stability of Multi-Agent systems in e.g. [38-40] and [41] respectively. As our approach is not restricted to passivity or $\mathcal{L}_{2}$-gain, the matrices $X, Y$ and $Z$ introduce extra degrees of freedom in order to reduce the conservatism. In the next subsection, a choice of these matrices is investigated.

\subsection{Proof of Corollary 1 and Theorem 1}

We first prove Corollary 1, which focuses on the stability, in order to afterwards prove Theorem 1 which focuses on the $H_{\infty}$ norm performance defined by (5).

Proof of Corollary 1 (stability). Note that due to the stability of $M$, the stability of

$$
\left(I_{N_{s}} \otimes T_{s}\right) \star M=M_{22}+M_{21}\left(I_{N_{s}} \otimes T_{s}\right)\left(I-M_{11}\left(I_{N_{s}} \otimes T_{s}\right)\right)^{-1} M_{12}
$$

is equivalent to the stability of $\left(I_{N_{s}} \otimes T_{s}\right)\left(I-M_{11}\left(I_{N_{s}} \otimes T_{s}\right)\right)^{-1}$, that is the feedback connection of $\left(I_{N_{s}} \otimes T_{s}\right)$ and $M_{11}$. The proof is based on the application of the separation of graph theorem presented in [27, Theorem 1] (see also [42]) which is presented here in a slightly different way.

Theorem 2. The negative feedback of a linear $\left\{-R,-S^{T},-Q\right\}$-dissipative operator $L$ and a static interconnection $H$ is stable if the following matrix is positive definite, i.e.:

$$
\widehat{Q}=S H+H^{T} S^{T}-H^{T} R H-Q>0
$$

Condition (i) of Corollary 1 states that the interconnection $M_{11}$ is $\{P \otimes X, P \otimes Y, P \otimes Z\}-$ dissipative. First let us prove that if condition (ii) of Corollary 1 holds $\left(T_{s}\right.$ is strictly $\left\{-Z,-Y^{T},-X\right\}$-dissipative) then for any positive definite matrix $P, I_{N_{s}} \otimes T_{s}$ is strictly 
$\left\{-P \otimes Z,-P \otimes Y^{T},-P \otimes X\right\}$-dissipative. Therefore the stability is deduced by applying Theorem 2 due to the fact that condition (i) of Corollary 1 implies condition (9) of Theorem 2 with $H=-M_{11}, L=I_{N} \otimes T_{s}$ and $Q=P \otimes X, S=P \otimes Y, R=P \otimes Z$.

Condition (ii) (the strict $\left\{-Z,-Y^{T},-X\right\}$-dissipativity of $T_{s}$ ) ensures that $\left(I_{N_{s}} \otimes T_{s}\right)$ is strictly $\left\{-I_{N_{s}} \otimes Z,-I_{N_{s}} \otimes Y^{T},-I_{N_{s}} \otimes X\right\}$-dissipative, i.e. for almost all $\omega \in \mathbb{R}$ and a real $\varepsilon_{1}>0$

$$
\begin{gathered}
{\left[\begin{array}{c}
I_{n_{s}} \\
T_{s}(j \omega)
\end{array}\right]^{*}\left[\begin{array}{cc}
-Z & -Y^{T} \\
-Y & -X
\end{array}\right]\left[\begin{array}{c}
I_{n_{s}} \\
T_{s}(j \omega)
\end{array}\right] \leq-\varepsilon_{1} I_{n_{s}}} \\
\Leftrightarrow\left[\begin{array}{c}
I_{N_{s}} \otimes T_{s}(j \omega) \\
I_{N_{s}} \otimes I_{n_{s}}
\end{array}\right]^{*}\left[\begin{array}{cc}
I_{N_{s}} \otimes X & I_{N_{s}} \otimes Y \\
I_{N_{s}} \otimes Y^{T} & I_{N_{s}} \otimes Z
\end{array}\right]\left[\begin{array}{c}
I_{N_{s}} \otimes T_{s}(j \omega) \\
I_{N_{s}} \otimes I_{n_{s}}
\end{array}\right] \geq \varepsilon_{1} I_{N_{s}} \otimes I_{n_{s}}
\end{gathered}
$$

By post- and pre-multiplying the previous inequality by an invertible full rank matrix $D \otimes I_{n_{s}}$ with $D \in \mathbb{R}^{N_{s} \times N_{s}}$ and its transpose, by noting that

$$
\left(I_{N_{s}} \otimes T_{s}(j \omega)\right) \times\left(D \otimes I_{n_{s}}\right)=\left(D \otimes I_{m_{s}}\right) \times\left(I_{N_{s}} \otimes T_{s}(j \omega)\right)
$$

and by using the properties of the Kronecker product [43], one obtains for almost all $\omega \in \mathbb{R}$

$$
\left[\begin{array}{c}
I_{N_{s}} \otimes T_{s}(j \omega) \\
I_{N_{s}} \otimes I_{n_{s}}
\end{array}\right]^{*}\left[\begin{array}{cc}
\left(D^{T} D \otimes I_{m_{s}}\right)\left(I_{N_{s}} \otimes X\right) & \left(D^{T} D \otimes I_{m_{s}}\right)\left(I_{N_{s}} \otimes Y\right) \\
\left(D^{T} D \otimes I_{n_{s}}\right)\left(I_{N_{s}} \otimes Y^{T}\right) & \left(D^{T} D \otimes I_{n_{s}}\right)\left(I_{N_{s}} \otimes Z\right)
\end{array}\right]\left[\begin{array}{c}
I_{N_{s}} \otimes T_{s}(j \omega) \\
I_{N_{s}} \otimes I_{n_{s}}
\end{array}\right] \geq \varepsilon_{1} D^{T} D \otimes I_{n_{s}}
$$

which for $P=D^{T} D>0$ is equivalent to for almost all $\omega \in \mathbb{R}$ a real $\varepsilon_{1}>0$

$$
\left[\begin{array}{c}
I_{N_{s}} \otimes T_{s}(j \omega) \\
I_{n_{s} \times N_{s}}
\end{array}\right]^{*}\left[\begin{array}{cc}
P \otimes X & P \otimes Y \\
P \otimes Y^{T} & P \otimes Z
\end{array}\right]\left[\begin{array}{c}
I_{N_{s}} \otimes T_{s}(j \omega) \\
I_{n_{s} \times N_{s}}
\end{array}\right] \geq \varepsilon_{1} P \otimes I_{n_{s}} \geq \varepsilon_{2} I_{n_{s} \times N_{s}}
$$

with new real $\varepsilon_{2}>0$ such that $\varepsilon_{2} \leq \varepsilon_{1} \bar{\sigma}\left(P \otimes I_{n_{s}}\right)=\varepsilon_{1} \bar{\sigma}(P)$. The last inequality implies that $I_{N_{s}} \otimes T_{s}$ is strictly $\left\{-P \otimes Z,-P \otimes Y^{T},-P \otimes X\right\}$-dissipative which concludes the proof. 
Proof of Theorem 1 (performance). Note that condition (i) and condition (ii) of Theorem 1 imply condition (i) and condition (ii) of Corollary 1, which ensures stability.

Let us now prove the inequality (5), that is the inequality on the $H_{\infty}$ norm. By definition, $\left\{\operatorname{diag}\left(P \otimes X,-\eta^{2} I\right), \operatorname{diag}(P \otimes Y, 0), \operatorname{diag}(P \otimes Z, I)\right\}-$ dissipativity of $M$ is equivalent to the following quadratic condition $\forall w, \forall \tau>0$ :

$$
\int_{0}^{\tau}\left[\begin{array}{c}
p(t) \\
w(t)
\end{array}\right]^{T}\left[\frac{I}{-}\left[\begin{array}{cc|cc}
P \otimes X & 0 & P \otimes Y & 0 \\
0 & -\eta^{2} I & 0 & 0 \\
\hline P \otimes Y^{T} & 0 & P \otimes Z & 0 \\
0 & 0 & 0 & I
\end{array}\right]\left[\begin{array}{c}
I \\
M
\end{array}\right]\left[\begin{array}{c}
p(t) \\
w(t)
\end{array}\right] d t \leq 0\right.
$$

From equation (1) (definition of the global system), one obtains:

$$
\begin{aligned}
& \int_{0}^{\tau}\left[\begin{array}{c}
z(t) \\
w(t)
\end{array}\right]^{T} \quad\left[\begin{array}{cc}
I & 0 \\
0 & -\eta^{2} I
\end{array}\right] \quad\left[\begin{array}{c}
z(t) \\
w(t)
\end{array}\right] d t \\
& \leq-\int_{0}^{\tau}\left[\begin{array}{l}
p(t) \\
q(t)
\end{array}\right]^{T}\left[\begin{array}{cc}
P \otimes X & P \otimes Y \\
P \otimes Y^{T} & P \otimes Z
\end{array}\right]\left[\begin{array}{l}
p(t) \\
q(t)
\end{array}\right] d t
\end{aligned}
$$

$\forall \tau>0, \forall w$ and $p, q, z$ defined by $(1)$.

The left hand part of (12) expresses the relation between the input $w$ and the output $z$ of the global system (1) while its right hand part is an integral quadratic constraints on the internal signals $q$ and $p$, that is, the input/output of $I_{N_{s}} \otimes T_{s}$ (see Fig.1). Since condition (11) ensures strict $\left\{-P \otimes Z,-P \otimes Y^{T},-P \otimes X\right\}$-dissipativity of $I_{N_{s}} \otimes T_{s}$, for some $\varepsilon>0$ and 
$\forall P=P^{T}>0$, with $p=I_{N_{s}} \otimes T_{s}(q)$, we have:

$$
\int_{0}^{\tau}\left[\begin{array}{c}
p(t) \\
q(t)
\end{array}\right]^{T}\left[\begin{array}{cc}
-P \otimes X & -P \otimes Y \\
-P \otimes Y^{T} & -P \otimes Z
\end{array}\right]\left[\begin{array}{l}
p(t) \\
q(t)
\end{array}\right] d t \leq-\varepsilon \int_{0}^{\tau} q(t)^{T} q(t) d t \leq 0
$$

From condition (13) and condition (12), one obtains

$$
\int_{0}^{\tau}\left[\begin{array}{c}
z(t) \\
w(t)
\end{array}\right]^{T}\left[\begin{array}{cc}
I & 0 \\
0 & -\eta^{2} I
\end{array}\right]\left[\begin{array}{l}
z(t) \\
w(t)
\end{array}\right] d t \leq 0
$$

$\forall \tau>0, \forall w$ and $z$ defined by (1).

Applying Fourier transform, the last equation becomes

$$
\int_{-\infty}^{+\infty}\left[\begin{array}{c}
z(j \omega) \\
w(j \omega)
\end{array}\right]^{*}\left[\begin{array}{cc}
I & 0 \\
0 & -\eta^{2} I
\end{array}\right]\left[\begin{array}{l}
z(j \omega) \\
w(j \omega)
\end{array}\right] d \omega \leq 0
$$

Leaving out the integral and noting that $z=\left(I_{N_{s}} \otimes T_{s}\right) \star M(w)$ the following condition on external input output signals in frequency domain is obtained: for almost $\forall \omega \in \mathbb{R}^{+}$

$$
\left[\begin{array}{c}
\left(I_{N_{s}} \otimes T_{s}\right) \star M(j \omega) \\
I(t)
\end{array}\right]^{*}\left[\begin{array}{cc}
I & 0 \\
0 & -\eta^{2} I
\end{array}\right]\left[\begin{array}{c}
\left(I_{N_{s}} \otimes T_{s}\right) \star M(j \omega) \\
I(t)
\end{array}\right] \leq 0
$$

which is equivalent to $\left\|\left(I_{N_{s}} \otimes T_{s}\right) \star M\right\|_{\infty} \leq \eta$, i.e. the inequality (5).

\subsection{How to choose $X, Y, Z$ ?}

In order to apply Theorem 1 and Corollary 1 , it is necessary to choose the matrices $X$, $Y$ and $Z$. For the sake of clarity, we focus on static interconnections, that is $M$ is a real matrix, with $n_{s}=1, m_{s}=1$, i.e. $X, Y, Z$ are scalars, referred to as in the sequel $x, y$ and 
$z$, and $T_{s}$ SISO. The case of a dynamical LTI interconnections $M$ can be investigated in a similar way. However, the choice of the matrices in the Multi Input Multi Output (MIMO) case is the perspective of a future work.

The main idea of the choice of $x, y$ and $z$ is to satisfy the condition (i) of Corollary 1 and Theorem 1 and to relax condition (ii) as much as possible. Indeed, it allows to obtain the least restrictive constraint $\|\widehat{T}\|_{\infty}<1($ see $(7))$ in order to compute the local controller by solving a standard $H_{\infty}$ problem. We first discuss the choice of $x, y$ and $z$ for the application of Corollary 1 and then for the application of Theorem 1.

\section{Conditions of Corollary 1 (Stability)}

Condition (ii) of Corollary 1 enforces the transfer function $T_{s}$ to be strictly $\{-z,-y,-x\}-$ dissipative, that is:

$$
\begin{aligned}
& {\left[\begin{array}{c}
T_{s}(j \omega) \\
1
\end{array}\right]^{*}\left[\begin{array}{ll}
x & y \\
y & z
\end{array}\right]\left[\begin{array}{c}
T_{s}(j \omega) \\
1
\end{array}\right]>0} \\
& \Longleftrightarrow\left(T_{s}(j \omega)-c\right)^{*}\left(T_{s}(j \omega)-c\right)<r^{2}
\end{aligned}
$$

with $c=-\frac{y}{x}$ and $r=\sqrt{\frac{y^{2}}{x^{2}}-\frac{z}{x}}$. The last inequality means that the Nyquist plot of the transfer function $T_{s}$ is inside the circle with center $c$ and radius $r$. Fig.2 presents the Nyquist plot of $T_{s}$ in the case of a typical PLL design (blue line), with the circle plotted in full red line. To relax the constraint (15) for a given center $c$, the radius $r$ has to be maximized. Note that the radius $r$ can be increased by increasing the positive $-\frac{z}{x}$. Similarly, condition (i) of Corollary 1 which enforces the matrix $M_{11}$ to be $\{x P, y P, z P\}$-dissipative with 


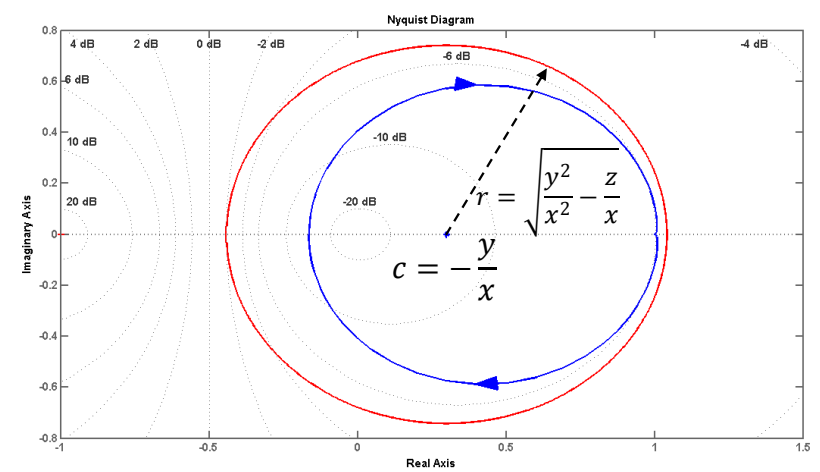

Figure 2: Typical Nyquist plot of a transfer function $T_{s}$

$P>0$, can be expressed as:

$$
\left(M_{11}+\frac{y}{z} I\right)^{T} P\left(M_{11}+\frac{y}{z} I\right) \leq\left(\frac{y^{2}}{x^{2}}-\frac{z}{x}\right) P
$$

Increasing $-\frac{z}{x}$ strengthens the constraint (16). Thus constraint (15) and constraint (16) cannot be simultaneously relaxed. The following optimization problem should therefore be solved: "find $x, y, z$ such that the condition (16) is satisfied and that it maximizes the circle radius $r$ for a given center $c "$.

To solve this problem, without lost of generality, one redundant variable can be eliminated by setting for example $z=1^{1}$. By introducing in condition (16) the variables $r$ and $c$, the

\footnotetext{
${ }^{1}$ This is equivalent to dividing both inequalities (14) and (16) by $z>0$ and performing the change of variables: $\tilde{x}=\frac{x}{z} \leq 0, \tilde{y}=\frac{y}{z}$.
} 
optimization problem can be formulated as:

$$
\min _{\chi, P} \quad \chi=\frac{1}{r^{2}}
$$

such that

$$
\begin{array}{lcc}
P & > & 0 \\
M_{11}^{T} P M_{11} \leq \underbrace{\frac{1}{r^{2}}}_{\chi}\left(c M_{11}-I\right)^{T} P\left(c M_{11}-I\right)
\end{array}
$$

For a given $c$, the minimization problem of $\chi$ (and thus the maximization of $r$ ) with the decision variables $P \in \mathbb{R}^{N_{s} \times N_{s}}$ and $\chi \in \mathbb{R}^{+}$, is a generalized eigenvalue problem involving Linear Matrix Inequality constraints, that is a standard (quasi convex) LMI optimization problem [37]. If $c$ is a decision variable, the optimization problem (17) is no longer a generalized eigenvalue minimization problem. However, based on the spectrum of the matrix $M_{11}, c$ can be chosen in order to ensure the feasibility of condition (16): with $\lambda_{i}^{M_{11}}$ is the $i$-th eigenvalue of $M_{11}$

$$
|c|=\left|\frac{z}{x}\right| \leq \frac{1}{2 \max _{i}\left(\left|\operatorname{Re}\left(\lambda_{i}^{M_{11}}\right)\right|\right)} .
$$

Then, using a linear search on $c$ in the interval defined by (18), the best $c$ and $r$ can be found in the sense of relaxing the $T_{s}$ transfer function circle constraint (15) and thus the condition (ii) of Corollary 1. Finally, $\{x, y, z=1\}$ is obtained from $\{r, c\}$ by a simple transformation. Let us now generalize this result to the global performance case. 


\subsection{Theorem 1 conditions (Performance)}

As in the previous subsection, condition (i) of Theorem 1 is equivalently expressed in terms of the center $c$ and the radius $r$ : there exists $P=P^{T}>0$ such that

$$
\left[\begin{array}{l}
I \\
\hline M
\end{array}\right]^{T}\left[\begin{array}{cc|cc}
-P & 0 & c P & 0 \\
0 & -\eta^{2} I & 0 & 0 \\
\hline c P & 0 & \left(r^{2}-c^{2}\right) P & 0 \\
0 & 0 & 0 & I
\end{array}\right]\left[\begin{array}{c}
I \\
\hline \\
M
\end{array}\right] \leq 0
$$

Condition (19) can be factorized as:

$$
\left[\begin{array}{l}
I \\
\hline M
\end{array}\right]^{T}\left[\begin{array}{cc|cc}
I & 0 & -c I & 0 \\
0 & I & 0 & 0 \\
\hline 0 & 0 & I & 0 \\
0 & 0 & 0 & I
\end{array}\right]^{T}\left[\begin{array}{cc|cc}
-P & 0 & 0 & 0 \\
0 & -\eta^{2} I & 0 & 0 \\
\hline 0 & 0 & r^{2} P & 0 \\
0 & 0 & 0 & I
\end{array}\right]\left[\begin{array}{cc|cc}
I & 0 & -c I & 0 \\
0 & I & 0 & 0 \\
\hline 0 & 0 & I & 0 \\
0 & 0 & 0 & I
\end{array}\right]\left[\begin{array}{c}
I \\
M
\end{array}\right] \leq 0
$$


With the change of variables $\widehat{P}=r^{2} P$ and $\beta=\eta^{2} r^{2}$, the following optimization problem is obtained:

$$
\min _{\chi, \widehat{P}} \quad \chi=\frac{1}{r^{2}}
$$

$$
\begin{gathered}
\widehat{P} \\
\text { such that } \Phi^{T}\left[\begin{array}{cc|cc}
0 & 0 & 0 & 0 \\
0 & 0 & 0 & 0 \\
\hline 0 & 0 & \widehat{P} & 0 \\
0 & 0 & 0 & I
\end{array}\right] \Phi \leq \underbrace{\frac{1}{r^{2}}}_{\chi} \Phi^{T}\left[\begin{array}{cc|cc}
\widehat{P} & 0 & 0 & 0 \\
0 & \beta I & 0 & 0 \\
\hline 0 & 0 & 0 & 0 \\
0 & 0 & 0 & 0
\end{array}\right] \Phi
\end{gathered}
$$

where $\Phi=\left[\begin{array}{cc|cc}I & 0 & -c I & 0 \\ 0 & I & 0 & 0 \\ \hline 0 & 0 & I & 0 \\ 0 & 0 & 0 & I\end{array}\right]\left[\begin{array}{c}I \\ M\end{array}\right]$.

The optimization problem (20) with a given center $c$ and ratio $\beta$ is a generalized eigenvalue problem involving Linear Matrix Inequality constraints.

Remark 4. The parameter $\beta=\eta^{2} / \chi$ is a tuning parameter for the condition (20). For a given $\beta$, the minimization of $\chi$ using (20) maximizes the radius $r$ of the circle and thus relaxes the constraint (15). Furthermore, the upper bound $\eta$ on the $H_{\infty}$-norm of the global performance transfer function i.e. $\left\|\left(I_{N_{s}} \otimes T_{s}\right) \star M\right\|_{\infty}$ is minimized. If the optimization problem (20) has no solution or the computed radius $r=1 / \sqrt{\chi}$ is too tight constraint on 
(15), $\beta$ should be increased to relax the condition of the optimization problem (20). The initial value of $\beta=r^{2} \eta^{2}$ can be computed for the control law $K$ which only ensures the global stability, i.e. the conditions of Corollary 1 for $x, y, z$ found by solving the optimization problem (17).

Remark 5. The result of the optimization problem (20) is both 1) a property of $\{x, y, z\}$ dissipativity to be ensured by the controller $K$ i.e. condition (ii) of Theorem 1 and 2) an upper bound on the global transfer function $H_{\infty}$-norm i.e. the performance objective. This upper bound is actually found before the computation of the control law. It a priori ensures a bound on the achieved global performance level if the controller ensures the local dissipativity constraint i.e. condition (ii) of Theorem 1.

\section{Local control for global performance}

When applying Theorem 1 or Corollary 1, if condition (i) is satisfied and in order to solve Problem 1 , it is necessary to find $K$ such that $T_{s}=G \star K$ is strictly $\left\{-Z,-Y^{T},-X\right\}-$ dissipative. As explained in the discussion page 13, this problem reduces to compute $K$ such that $\|\hat{T}\|_{\infty}<1$ where $\hat{T}$ is defined by (7), which is a standard $H_{\infty}$ problem.

As discussed in the previous sections, in the Microelectronics design of PLL networks, as $T_{s}$ represents a PLL, the design of $K$ such that $T_{s}=G \star K$ satisfies some specifications, corresponds to the local design step. A strong interest of Theorem 1 or Corollary 1 is to reveal how to design the controller $K$ of the local system $T_{s}=G \star K$ in order to a priori ensure the stability of the global system $\left(I_{N_{s}} \otimes T_{s}\right) \star M$. Theorem 1 also guarantees the 
global system performance defined by (2) and thus present a solution to Problem 1 . The question is to investigate how to apply this result in order to directly enforce typical design specifications on the global system at the local design step.

A popular approach to specify the performance of a (closed-loop) system is to define frequency dependent upper bound on the magnitude of the frequency response of several (closed-loop) Single Input, Single Output (SISO) transfer functions, see e.g. [19,44,45]. The computation of the controller such that the performance is satisfied can be expressed as an optimization problem involving an inequality on the weighted $H_{\infty}$ norm of the closed loop system. Such a performance specification is particularly interesting in Microelectronics [4649]. As a consequence, the computation of the filter $K$ of a PLL can be expressed as a standard $H_{\infty}$ control problem: compute $K$ such that

$$
\left\|W_{l p o} G_{l p} \star K W_{l p i}\right\|_{\infty}<1
$$

where $G_{l p} \star K$ is a matrix of transfer functions which includes the closed-loop transfer functions associated to the performance and where $W_{l p o}=\operatorname{diag}_{i}\left(w_{l p o}^{i}\right)$ and $W_{l p i}=\operatorname{diag}_{j}\left(w_{l p i}^{j}\right)$ are diagonal matrices of weighting functions. The inequality (21) ensures that the magnitude of the frequency response of the transfer function between the input $j$ and the output $i$ is below $\frac{1}{\left|w_{l p o}^{i}(j \omega)\right|\left|w_{l p i}^{j}(j \omega)\right|}$ for all frequency $\omega$.

In the following theorem, we reveal how to enforce, with a good choice of $G_{l p}$, a given frequency dependent upper bound on the magnitude of the frequency response of the global system transfer function i.e. on $\left|\left(I_{N_{s}} \otimes T_{s}(j \omega)\right) \star M\right|$, by computing a controller $K$ such that (21). To do so, a new global weighting function $W=\left(I_{N_{w}} \otimes T_{s}\right) \star M_{W}$ will be used. 
Please notice that this transfer function is chosen as the global system (1) to be in the form of interconnection of $N_{w}$ subsystems $T_{s}$ and static matrix $M_{W}$. The reason of such choice will be discussed after theorem. For the sake of clearness, the result is presented in the case of one SISO global transfer function, i.e. $n_{z}=n_{w}=1$ : nevertheless, due to the MIMO $H_{\infty}$ norm constraint properties [19], the result can be applied in the case of several ones i.e. for MIMO global transfer functions.

Theorem 3. Let be $\eta>0$, a stable interconnection $M$, 3 real matrices $X=X^{T} \in \mathbb{R}^{m_{s} \times m_{s}} \geq$ $0, Y \in \mathbb{R}^{m_{s} \times n_{s}}, Z=Z^{T} \in \mathbb{R}^{n_{s} \times n_{s}}, G$ and $G_{l p}$ which define the local subsystem $T_{s}$ and a SISO local performance (lp) transfer function $T_{l p}$ such that:

- $G \star K=T_{s}$

- $G_{l p} \star K=T_{l p}$

and a SISO stable interconnected system $W$ defined by $\left(I_{N_{w}} \otimes T_{s}\right) \star M_{W}$ such that

$$
|W(j \omega)|^{-1} \approx\left|T_{l p}(j \omega)\right|
$$

If there exists

(i) a positive definite matrix $P \in \mathbb{R}^{\left(N_{s}+N_{w}\right) \times\left(N_{s}+N_{w}\right)}$ such that $\widetilde{M}$ is $\left\{\operatorname{diag}\left(P \otimes X,-\eta^{2} I\right)\right.$, $\operatorname{diag}(P \otimes Y, 0), \operatorname{diag}(P \otimes Z, I)\}-$ dissipative where $\widetilde{M}$ is defined by:

$$
\left(I_{N_{s}+N_{w}} \otimes T_{s}\right) \star \widetilde{M}=\left(I_{N_{w}} \otimes T_{s}\right) \star M_{W} \times\left(I_{N_{s}} \otimes T_{s}\right) \star M
$$

(ii) a controller $K$ such that 


$$
\begin{aligned}
& \left\|(-X)^{\frac{1}{2}}\left(G+X^{-1} Y\right) \star K\left(Z-Y^{T} X^{-1} Y\right)^{-\frac{1}{2}}\right\|_{\infty}<1 \\
& \left\|W_{l p o} G_{l p} \star K W_{l p i}\right\|_{\infty} \quad<1
\end{aligned}
$$

then the controller $K$ ensures that the global system $\left(I_{N_{s}} \otimes T_{s}\right) \star M$ is stable and

$$
\left|\left(\left(I_{N_{s}} \otimes T_{s}\right) \star M\right)(j \omega)\right|_{d B} \leq-\left|W_{l p o}(j \omega) W_{l p i}(j \omega)\right|_{d B}+20 \log _{10}(\eta), \quad \forall \omega
$$

Proof. This theorem is proved by applying Theorem 1 to the (augmented) interconnected system $\left(I_{N_{s}+N_{w}} \otimes T_{s}\right) \star \widetilde{M}$ where $\widetilde{M}$ defined by $(22)$.

Let us assume that both conditions of this theorem are satisfied. Condition (i) is exactly condition (i) of Theorem 1. The first condition in (23) implies that there exist a controller $K$ such that

$$
\|\hat{T}\|_{\infty}<1
$$

where $\hat{T}$ is defined by (7). From the discussion on page 13 , this condition is equivalent to condition (ii) of Theorem 1. Then, by applying Theorem 1, the augmented system $\left(I_{N_{s}+N_{w}} \otimes T_{s}\right) \star \widetilde{M}$ is stable and

$$
\left\|\left(I_{N_{s}+N_{w}} \otimes T_{s}\right) \star \widetilde{M}\right\|_{\infty} \leq \eta
$$

As $W$ and $\left(I_{N_{s}} \otimes T_{s}\right) \star M$ are SISO, the last inequality is equivalent to:

$$
\left|\left(\left(I_{N_{s}} \otimes T_{s}\right) \star M\right)(j \omega)\right| \leq \frac{\eta}{|W(j \omega)|}, \quad \forall \omega .
$$

On the other hand, the second condition in (23) implies that (see the definition of $G_{l p}$ ):

$$
\left|T_{l p}(j \omega)\right|<\frac{1}{\left|W_{l p o}(j \omega) W_{l p i}(j \omega)\right|}
$$


Since $|W(j \omega)|^{-1} \approx\left|T_{l p}(j \omega)\right|$, we obtain:

$$
\left|\left(\left(I_{N_{s}} \otimes T_{s}\right) \star M\right)(j \omega)\right| \leq \frac{\eta}{\left|W_{l p o}(j \omega) W_{l p i}(j \omega)\right|}, \quad \forall \omega
$$

Inequality (24) is obtained by expressing the previous equation in $\mathrm{dB}$, which concludes the proof.

Remark 6 . The stable interconnected system $W$ can be interpreted as the global weighting function imposing a global performance frequency constraint as in the standard $H_{\infty}$ approach:

$$
\left\|W\left(\left(I_{N_{s}} \otimes T_{s}\right) \star M\right)\right\|_{\infty} \leq \eta
$$

Traditionally, in the $H_{\infty}$ framework, the system to be constrained is represented as an interconnection of integrators i.e. a state-space representation. It is also the case for the weighting functions as well and then for the augmented system. The existing efficient algorithms of the standard $H_{\infty}$ problem resolution (see e.g. $[19,37]$ ) are based on this important fact.

Nevertheless, in our problem, the global system $\left(I_{N_{s}} \otimes T_{s}\right) \star M$ is naturally represented as an interconnection of the subsystems dynamics $T_{s}$ (see (1) and Fig. 1). For this reason, the weighting function $W$, similarly to the standard $H_{\infty}$ framework, has to be represented as the interconnection of $N_{w}$ subsystems $T_{s}$.

Another requirement on the weighting function $W$ is that its magnitude should be equal to the magnitude of the inverse of a local performance transfer function, at least for some frequency ranges of interest. This implies that the local performance transfer function $T_{l p}$ should be chosen as well as an interconnection of $T_{s}$. This original idea allows to introduce 
a natural and strong connection between the local $T_{l p}$ and global $\left(I_{N} \otimes T_{s}\right) \star M$ performance transfer functions so that their difference can be easily expressed and minimized.

Remark 7 . The proof of Theorem 3 reveals that the inequality (24) is deduced from the inequality:

$$
\left|\left(\left(I_{N} \otimes T_{s}\right) \star M\right)(j \omega)\right|_{d B} \leq\left|T_{l p}(j \omega)\right|_{d B}+20 \log _{10}(\eta), \quad \forall \omega
$$

The inequality (27) presents the relation between the shape of the frequency response of the global system $\left(I_{N} \otimes T_{s}\right) \star M$, that is the global performance, and the shape of the frequency response of the stand-alone subsystem $T_{l p}$ that is the local performance.

Discussion In Theorem 3, the first inequality of (23) ensures the strict $\left\{-Z,-Y^{T},-X\right\}$ dissipativity of $T_{s}$ which is necessary to the application of Theorem 1 . The second inequality ensures the local subsystem performance i.e. the performance of an independent stand-alone subsystem $T_{l p}$ which is expressed as the upper frequency bound (26). Therefore, since the stable interconnected system $W$ is defined as the inverse of $T_{l p}$ expressed as an interconnection of $T_{s}$, its series combination with the global system (22) defines an augmented system describing a relation between the global and local performance transfer functions. Ensuring a bound $\eta$ on this relation allows to ensure the global system performance (24) by enforcing the local subsystem performance (26).

Theorem 3 allows to compute the local controller $K$ that ensures the global system performance expressed in the form of a frequency upper bound (24). This computation is performed in some how relative fashion i.e. the global system performance is rather ensured in the relative then the absolute way (see Remark 7). We now discuss how it is possible 
to transform the global system performance problem into a standard local $H_{\infty}$ controller design problem that implies (23).

Assume that the condition (i) of the Theorem 3 is satisfied. The computation of the controller such that both inequalities in (23) is actually a multi-objective $H_{\infty}$ optimization problem. It is usually transformed into a standard one objective optimization problem by defining an augmented plant $\widetilde{G}$ such that, for example:

$$
\begin{gathered}
\bullet\left[\begin{array}{ll}
I & 0
\end{array}\right] \widetilde{G} \star K\left[\begin{array}{l}
I \\
0
\end{array}\right]=G \star K=T_{s} \\
\text { - }\left[\begin{array}{ll}
0 & I
\end{array}\right] \widetilde{G} \star K\left[\begin{array}{l}
0 \\
I
\end{array}\right]=G_{l p} \star K=T_{l p}
\end{gathered}
$$

The standard $H_{\infty}$ problem can now be defined: find the controller $\mathrm{K}$ such that:

$$
\left\|\operatorname{diag}\left((-X)^{\frac{1}{2}}, W_{l p o}\right)\left(\left(\widetilde{G}+\operatorname{diag}\left(X^{-1} Y, 0,0\right)\right) \star K\right) \operatorname{diag}\left(\left(Z-Y^{T} X^{-1} Y\right)^{-\frac{1}{2}}, W_{l p i}\right)\right\|_{\infty}<1 .
$$

Due to the $H_{\infty}$ norm properties, the controller $K$ such that the inequality (28) is satisfied is such that the inequalities (23) are satisfied. Depending on the problem under consideration, it could be more interesting to replace the $H_{\infty}$ problem defined by $(28)$ by another $H_{\infty}$ problem :

$$
\left\|W^{o} \widehat{G} \star K W^{i}\right\|_{\infty}<1
$$

where the choice of the plant $\widehat{G}$ and the weighting functions $W^{o}$ and $W^{i}$ is different from the choice presented in (28) but where the inequality (29) implies the inequalities (23). The motivation of this idea is illustrated on the numerical example presented in the next section. 


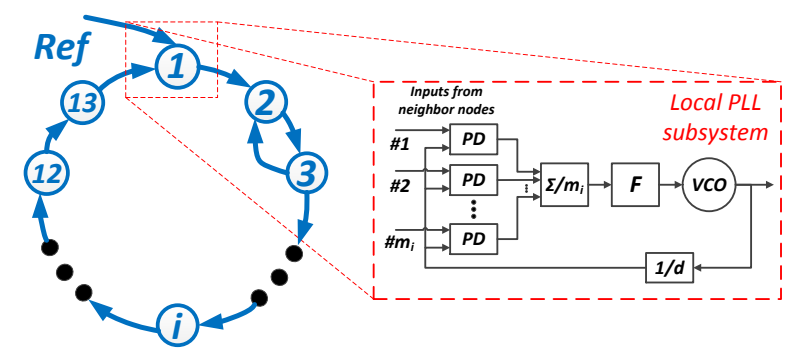

Figure 3: The active clock distribution network

\section{PLL Network design}

In this section, the method presented in Section 3 is applied to the design of an active clock distribution network, that is, a network of $N_{s}$ Phase-Locked Loops (PLL), (see Fig. 3). A Phase-Locked Loop is a feedback system that generates a periodic output signal which has to be synchronized (in frequency and/or in phase) with an external periodic signal. Each PLL of the network is composed of a Voltage Controlled Oscillator $(V C O)$ generating a local periodic signal which is fed back through a frequency divider $(1 / d)$ to the Phase Detectors $(P D)$. The purpose of each $P D$ is to measure the phase difference between the $V C O$ signal and the $m_{i}$ external input signals, up to a scaling factor, see Fig. 3. From the average of the $m_{i}$ differences (block $\Sigma / m_{i}$ ), the Filter $(F)$ computes the command signal which is the input of the $V C O$ and should modify the frequency of its output. In a network of PLLs, the $P D$ s between adjacent PLLs are usually factorized.

The Microelectronics design problem is to design an active clock distribution network (an example is presented Fig.3) that achieves the frequency and phase synchronization of the $N_{s}$ VCO outputs to an external reference signal, denoted $R e f$, within a specified time and 
with a suitable damping. Since the main parameters of a $V C O$ and a $P D$ as well as the network topology are deduced from the technology process, the chip structure and the clock generation requirements (clock frequency, number of PLLs with their localization, $V C O$ and $P D$ resolution, etc.), the design problem can be expressed as the design of the filter $F$ such that the active clock network achieves the specifications.

In the example detailed below, we focus on a network of $N_{s}=13$ PLLs with a circular topology and one external reference signal connected to the PLL 1, see Fig.3. Such an example is challenging since the traditional design procedure fails to achieve the design of a satisfactory network, see [50]. This point is emphasized in the next subsection with the application of the traditional design procedure. The application of our approach is investigated in the subsection 4.2 and the subsection 4.3 .

\subsection{Traditional design process}

The traditional design process consists in: (i) design a filter $F$ such that the stand-alone PLL has a good behavior and (ii) evaluation of the behavior of the active clock distribution network with this PLL. The design of a stand-alone PLL filter is based on a model where the periodic signals are represented by their phase $[47,49,51]$. In this domain, the synchronization of the $V C O$ signal with a periodic (sinusoidal) external signal is expressed as tracking the phase of the $V C O$ signal given by a ramp whose slope is the reference oscillator frequency. In addition, the phase domain PLL model, which is non-linear, can be 


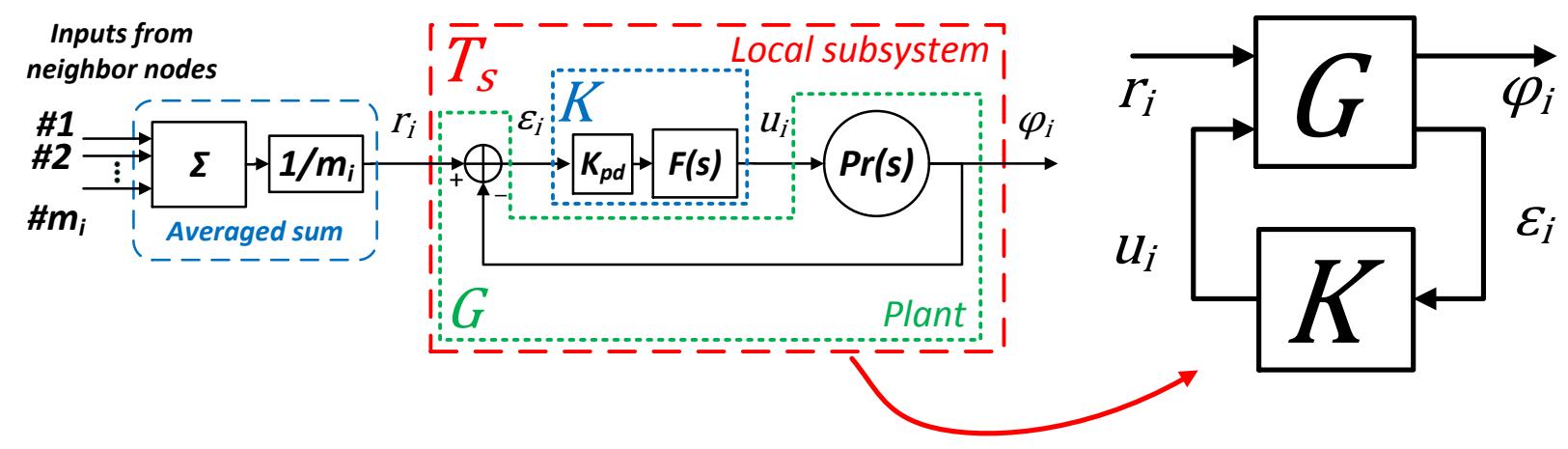

Figure 4: Phase domain model of the PLL subsystem

linearized around an operating point ${ }^{2}$. Based on this assumption and the PLL representation illustrated in Fig.3, the (linearized phase domain) model of one PLL is presented in Fig.4 (left part), where $K_{p d}$ denotes the phase detector linear gain and $\operatorname{Pr}(s)$ the $\operatorname{VCO}$ transfer function defined with $K_{V C O}$ the gain of the $V C O$ by

$$
\operatorname{Pr}(s)=\frac{K_{V C O} / d}{s}
$$

The numerical values are presented in Tab. 1 where the $V C O$ central frequencies represent constant perturbations at the $V C O$ inputs that have to be rejected to ensure the synchronization.

Using the traditional frequency domain design methods, the following filter $F$ is obtained with a satisfying behavior (PLL synchronization with periodical external signal at its input, response time less then $6 \mu s e c$, infinite gain margin, phase margin of 79 degrees, etc.):

$$
F(s)=0.44 \frac{s+464.2}{s}
$$

\footnotetext{
${ }^{2}$ One major non-linear issue of such PLL network known as mode-locking states will not be addressed in this paper but can be circumvented independently as in $[2,3,5]$.
} 
Table 1: Numerical values of the considered clock distribution network

\begin{tabular}{|c|c|}
\hline Parameter & Numerical value \\
\hline \hline Reference frequency & $f_{\text {ref }}=50 \mathrm{kHz}$ \\
\hline Frequency divider factor & $d=4$ \\
\hline$V C O$ central frequencies & random initialized \\
& around $d \cdot f_{\text {ref }} \pm 25 \%$ \\
\hline$V C O$ gain & $K_{V C O}=160.12 \mathrm{~Hz} / \mathrm{c} . u$ \\
\hline$P D$ gain & $K_{P D}=21.22$ e.u. $/ \mathrm{rad}$ \\
\hline Number of inputs & $m_{i} \in\{1,2\}$ depending \\
& on PLL position \\
\hline Dimensions & $N_{s}=13, n_{s}=1, m_{s}=1$ \\
\hline
\end{tabular}




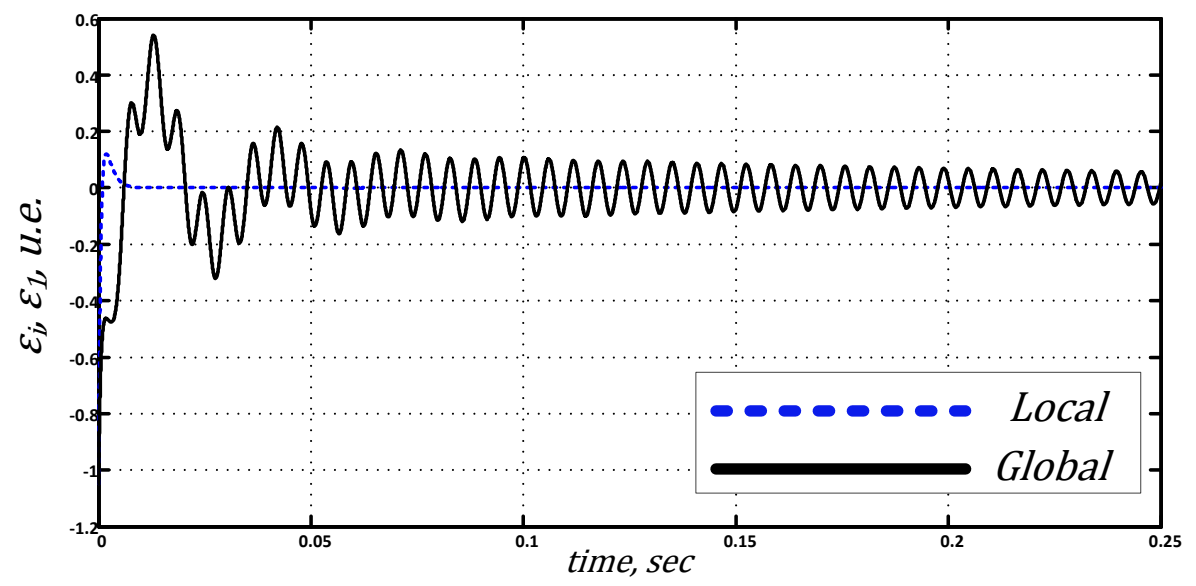

Figure 5: Synchronization errors of a stand-alone PLL (dashed blue line) and of PLL 1 of the global network (full black line)

Unfortunately, the obtained active clock distribution network does not behave as well as one stand-alone PLL, see Fig.5. In this figure, the time-domain simulation reveals that, in contrast with the synchronization error $\varepsilon_{i}$ of the stand-alone PLL (blue line), the synchronization error $\varepsilon_{1}$ of the PLL 1 of the network is not well-damped (considerable overshoot and parasite oscillations) and slowly decays to zero (around 100 times). This different behavior can be observed in the frequency domain. Let $S_{l o c}=T_{r_{i} \rightarrow \varepsilon_{i}}$ be the transfer function between the reference input $r_{i}$ and the synchronization error $\varepsilon_{i}=r_{i}-\varphi_{i}$ of the stand-alone PLL, see Fig. 4:

$$
S_{l o c}(s)=\frac{1}{1+F(s) K_{P D} \operatorname{Pr}(s)}
$$

Let $S_{g l o b}=T_{R e f \rightarrow \varepsilon_{1}}$ be the transfer function between the reference input Ref of the network and the PLL 1 synchronization error defined as the difference between the reference signal and the PLL 1 VCO output: $\varepsilon_{1}=\operatorname{Ref}-\varphi_{1}$. Fig.6 illustrates the magnitude of the frequency response of $S_{l o c}$ (dashed blue line) and of $S_{g l o b}$ (full blue line). In both cases, 


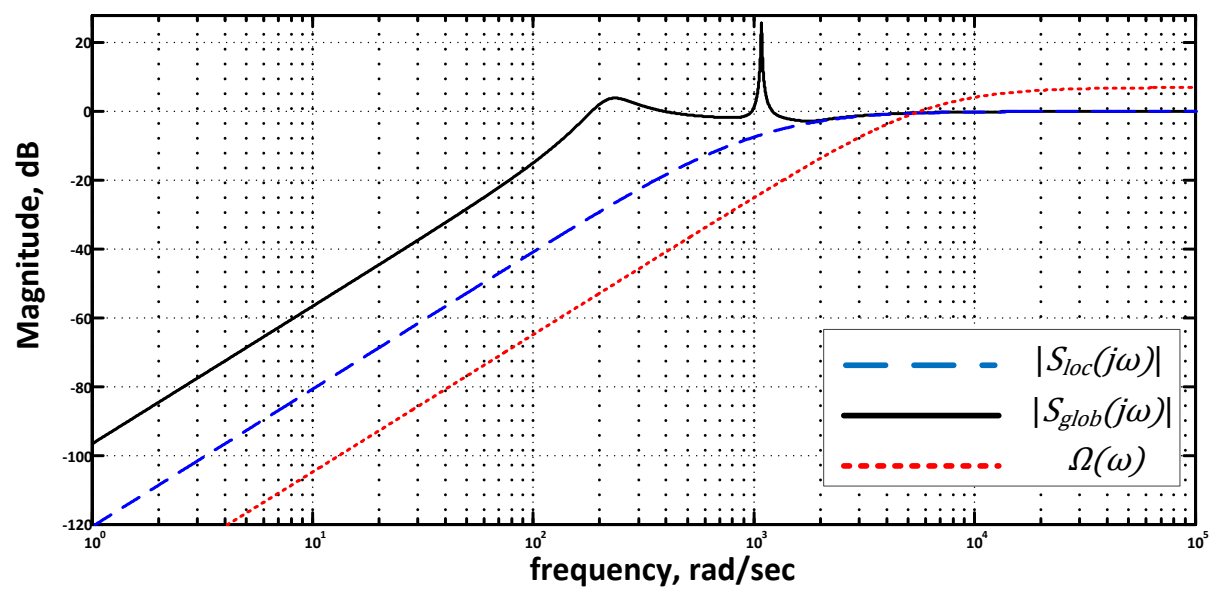

Figure 6: Magnitude of the transfer functions $S_{l o c}$ (dashed blue line) and $S_{g l o b}$ (full black line), as well as the considered frequency constraint (red dotted line) ensuring the global performance

the synchronization (or ramp tracking) is ensured by the slope of $+40 \mathrm{~dB} / \mathrm{dec}$ in the low frequency (LF) range. The time response is reduced by increasing the cut-off frequency and decreasing the transfer function magnitude in the LF range. The overshoot is limited by reducing the maximal transfer function gain (see $[19,52]$ for more details). Therefore, in order to improve the behavior of the PLL network (reduced time response and welldampedness), the magnitude of the frequency response of the transfer function $S_{g l o b}$ has to respect the frequency constraint represented by the red dotted line in Fig.6. This frequency constraint enforces a more important cut-off frequency and magnitude attenuation in LF range while maintaining the peak low in the middle frequency range. It can be represented as the magnitude of the frequency response $\Omega(\omega)$ of a transfer function as defined below:

$$
\Omega(s)=\left|W_{g l}(s)\right|^{-1} /\left(W_{g l}(s)\right)^{-1}=\frac{2.23(s+0.56)^{2}}{(s+6269)^{2}} .
$$


In the next subsection, we reveal how to design $F$ such that the magnitude of the frequency response of the transfer function $S_{g l o b}$ satisfies this frequency constraint.

\subsection{Proposed design process}

The purpose is to compute $F$ in order to enforce the synchronization of PLL 1 of the network with a specified behavior i.e. the frequency response of the global transfer function $S_{\text {glob }}$ respects the frequency constraint enforced by (30). To this purpose, the approach presented section 3 (Theorem 3) is iteratively applied by modifying $X, Y, Z$ and the weighting functions $W_{l p i}$ and $W_{l p o}$ until the global specifications are satisfied. The synchronization of all $N_{s}$ PLLs or more complex synchronization specifications [50] can be explicitly investigated by this approach. Nevertheless, for the sake of clarity, these cases are not presented in this example.

Notations First, let us redefine the problem in Theorem 3 notations. In the example under consideration, the transfer function $S_{g l o b}=T_{R e f \rightarrow \varepsilon_{1}}(s)$ can be represented as an 
interconnection $M$ of $N_{s}$ subsystems $T_{s}=G \star K,\left(I_{N} \otimes T_{s}(s)\right) \star M$, with

$$
\begin{aligned}
& M=\left[\begin{array}{ccccccc|c}
0 & 0 & 0 & 0 & \cdots & 0 & \frac{1}{2} & \frac{1}{2} \\
\frac{1}{2} & 0 & \frac{1}{2} & 0 & \cdots & 0 & 0 & 0 \\
0 & 1 & 0 & 0 & \cdots & 0 & 0 & 0 \\
\vdots & \ddots & \ddots & \ddots & \ddots & \vdots & \vdots & \vdots \\
\vdots & \ddots & \ddots & \ddots & \ddots & \vdots & \vdots & \vdots \\
0 & \ddots & \ddots & \ddots & \ddots & 0 & 0 & 0 \\
0 & \cdots & \cdots & \cdots & 0 & 1 & 0 & 0 \\
\hline-1 & 0 & \cdots & \cdots & \cdots & \cdots & 0 & 1
\end{array}\right] \\
& G(s)=\left[\begin{array}{rr}
0 & \operatorname{Pr}(s) \\
1 & -\operatorname{Pr}(s)
\end{array}\right] \text { and } K(s)=K_{p d} F(s) .
\end{aligned}
$$

The local performance transfer function $T_{l p}$ is defined as:

$$
T_{l p}(s)=S_{l o c}(s)=T_{r_{i} \rightarrow \varepsilon_{i}}(s)=\frac{1}{1+K(s) \operatorname{Pr}(s)}
$$

Thus, $T_{l p}=G_{l p} \star K$ with

$$
G_{l p}(s)=\left[\begin{array}{cc}
1 & -\operatorname{Pr}(s) \\
1 & -\operatorname{Pr}(s)
\end{array}\right] .
$$

Note that in our problem, $T_{s}$ is defined as $T_{s}(s)=\frac{K(s) \operatorname{Pr}(s)}{1+K(s) \operatorname{Pr}(s)} \cdot T_{l p}$ can be represented as an interconnection of $T_{s}$, since

$$
T_{l p}=1-T_{s}
$$

In order to ensure that $|W(j \omega)|^{-1} \approx\left|T_{l p}(j \omega)\right|$ for the frequency range of interest, where $W$ 


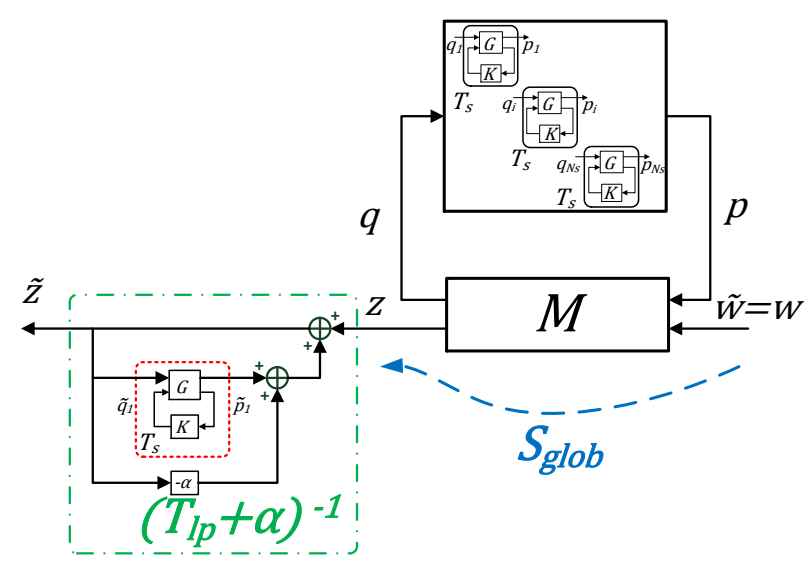

Figure 7: Augmented system $\left(I_{N_{s}+N_{w}} \otimes T_{s}\right) \star \widetilde{M}$

can be represented as an interconnection of subsystems $T_{s}$, we choose:

$$
W=\frac{1}{T_{l p}+\alpha}=\frac{1}{1+\alpha-T_{s}}
$$

where $\alpha=0.01$ is such that $W$ is well-posed ${ }^{3}$, stable and $|W(j \omega)|^{-1} \approx\left|T_{l p}(j \omega)\right|$ in the frequency range of interest. Then, $W=T_{s} \star M_{W}$ with

$$
M_{W}=\left[\begin{array}{cc}
(1+\alpha)^{-1} & (1+\alpha)^{-1} \\
(1+\alpha)^{-1} & (1+\alpha)^{-1}
\end{array}\right]=\left[\begin{array}{ll}
M_{11}^{W} & M_{12}^{W} \\
M_{21}^{W} & M_{22}^{W}
\end{array}\right]
$$

The system $S_{\text {glob }}$ augmented with the weighting function $W$ defined by $\left(I_{N+N_{w}} \otimes T_{s}\right) \star \widetilde{M}=$ $\left(I_{N_{w}} \otimes T_{s}\right) \star M_{W} \times\left(I_{N} \otimes T_{s}\right) \star M$ is represented in Fig. 7 while $\widetilde{M}$ is defined by:

$$
\widetilde{M}=\left[\begin{array}{ccc}
M_{11}^{W} & M_{12}^{W} M_{21} & M_{12}^{W} M_{22} \\
0 & M_{11} & M_{12} \\
M_{21}^{W} & M_{22}^{W} M_{21} & M_{22}^{W} M_{22}
\end{array}\right] .
$$

\footnotetext{
${ }^{3}$ For more details, see [53].
} 
Local control for global performance In order to ensure the global performance, by applying Theorem 3, the conditions (i) and (ii) should be ensured for some $X, Y, Z$ and $W_{l p}^{i}, W_{l p}^{o}$ chosen such that the frequency constraint defined by (30) and:

$$
-\left|W_{l p i}(j \omega) W_{l p o}(j \omega)\right|_{d B}+20 \log _{10}(\eta) \leq-20 \log (\Omega(\omega))
$$

Condition (i) of Theorem 3 is satisfied by an appropriate choice of $X, Y, Z$ which are scalars in the considered example. One possible choice is described in Subsection 2.4.

Condition (ii) (i.e. (23)) can be satisfied by solving the standard $H_{\infty}$ problem (28) with

$$
\widetilde{G}(s)=\left[\begin{array}{ccc}
0 & 0 & \operatorname{Pr}(s) \\
1 & 1 & -\operatorname{Pr}(s) \\
1 & 1 & -\operatorname{Pr}(s)
\end{array}\right]: \widetilde{G}(s) \star K=\left[\begin{array}{cc}
T_{s}(s) & T_{s}(s) \\
T_{l p}(s) & T_{l p}(s)
\end{array}\right] .
$$

Nevertheless, in order to ensure additional design specification on the PLL, the $H_{\infty}$ problem (28) is replaced by another $H_{\infty}$ problem (29).

Additional local performance specifications In addition to synchronization, the effects of the input/output $P D / V C O$ noises and possible perturbations have to be attenuated/rejected at the local level. The temperature and power disturbances as well as the central frequency variations of the $V C O$ can be modeled as a constant or a slowly varying perturbation on the $V C O$ input. Another specification is the limitation of the controller bandwidth (reduced control energy and noise attenuation). Such specifications can be enforced for a stand-alone PLL by ensuring upper bounds on the magnitude of the frequency response of the following transfer functions: 
- For synchronization, PLL damping, VCO output noise rejection: $T_{r_{i} \rightarrow \varepsilon_{i}}(s)=T_{l p}(s)$

- For $V C O$ input noise and constant perturbation rejection: $\operatorname{Pr}(s) T_{l p}(s)$

- For controller bandwidth limitation: $K(s) T_{l p}(s)$

- For $P D$ and reference signal noise rejection: $\operatorname{Pr}(s) K(s) T_{l p}(s)=T_{s}(s)$

Note that the first local performance specification is related to the transfer function $T_{l p}$, i.e. the same transfer function introduced to enforce the global performance. In order to take into account these local performance requirements, the $H_{\infty}$ problem (29) is solved with

$$
\widehat{G}=\left[\begin{array}{ccc}
1 & -\operatorname{Pr}(s) & -\operatorname{Pr}(s) \\
0 & 0 & 1 \\
X^{-1} Y & \operatorname{Pr}(s) & \operatorname{Pr}(s) \\
1 & -\operatorname{Pr}(s) & -\operatorname{Pr}(s)
\end{array}\right] \quad W^{i}=\operatorname{diag}\left(W_{1}^{i}, W_{2}^{i}\right)
$$

where $b=(-X)^{-\frac{1}{2}}\left(Z-Y^{T} X^{-1} Y\right)^{-\frac{1}{2}}$. Due to the properties of the $H_{\infty}$ norm, the solution of problem (29) directly implies the following conditions (see the expression of $\widehat{G}$ ).

$$
\left\|\left(T_{s}+X^{-1} Y\right) b\right\|_{\infty}<1
$$

that is, the first condition (23) of Theorem 3, and

$$
\begin{aligned}
& \left\|W_{1}^{o} \operatorname{Pr} T_{l p} W_{2}^{i}\right\|_{\infty}<1 \quad\left\|W_{2}^{o} K T_{l p} W_{1}^{i}\right\|_{\infty}<1 \\
& \left\|W_{2}^{o} \operatorname{Pr} K T_{l p} W_{2}^{i}\right\|_{\infty}<1 \quad\left\|W_{1}^{o} T_{l p} W_{1}^{i}\right\|_{\infty}<1
\end{aligned}
$$

The weighting functions $W_{1(2)}^{i(o)}$ are chosen such that they imply the local performance constraint i.e. the second constraint in (23) of Theorem 3 and the additional local performance specifications. 
It is true that the additional local performance is ensured for a stand-alone PLL and is not necessarily ensured for the PLL in the network (globally). Nevertheless, for some performance specification, global performance specification can be related to the local one. Let us focus on

- Reference noise rejection on the first PLL output: $T_{R e f \rightarrow \varphi_{1}}(s)=\operatorname{Pr}(s) K(s) S_{\text {glob }}(s)$

- Power consumption of the first PLL or, equivalently, the global bandwidth limitation of the first PLL : $T_{R e f \rightarrow u_{1}}(s)=K(s) S_{g l o b}(s)$

Note that

$$
\left|\frac{K(j \omega) S_{g l o b}(j \omega)}{K(j \omega) T_{l p}(j \omega)}\right|=\left|\frac{\operatorname{Pr}(j \omega) K(j \omega) S_{g l o b}(j \omega)}{\operatorname{Pr}(j \omega) K(j \omega) T_{l p}(j \omega)}\right|=\left|\frac{S_{g l o b}(j \omega)}{T_{l p}(j \omega)}\right|=\left|\frac{\left(\left(I_{N} \otimes T_{s}\right) \star M\right)(j \omega)}{T_{l p}(j \omega)}\right|
$$

From the proof of Theorem 3, if its conditions are satisfied then

$$
\left|\frac{\left(\left(I_{N} \otimes T_{s}\right) \star M\right)(j \omega)}{T_{l p}(j \omega)}\right| \leq \eta
$$

Therefore

$$
\left|T_{R e f \rightarrow u_{1}}(j \omega)\right|_{d B} \leq-\left|K(j \omega) T_{l p}(j \omega)\right|_{d B}+20 \log _{10}(\eta)
$$

and

$$
\left|T_{R e f \rightarrow \varphi_{1}}(j \omega)\right|_{d B} \leq-\left|\operatorname{Pr}(j \omega) K(j \omega) T_{l p}(j \omega)\right|_{d B}+20 \log _{10}(\eta)
$$

that is, for these specifications, the global performance specification is constrained by the local one. 


\subsection{Computation results}

The numerical results for the proposed design approach are now presented. On the iterations on the matrices $X, Y, Z$ and the weighting functions, we detail the numerical results for the last iteration, except for some graphical representation which can be present some intermediate results.

We first compute a filter $F$ such that the global system is stable by applying Corollary 1 where condition (ii) is replaced by the resolution of the $H_{\infty}$ problem defined by the first inequality of (23). In order to obtain the matrices $X, Y$ and $Z$ such that condition (i) of Corollary 1 is satisfied, the optimization problem (17) is solved. The circle center $c=$ 0.1 satisfies condition (18) since $\frac{1}{2 \max _{i}\left(\left|\operatorname{Re}\left(\lambda_{i}^{M_{11}}\right)\right|\right)}=0.5233$. Then the resolution of the optimization problem (17) gives $r=0.947, X=-1.129, Y=0.113, Z=1$. For these values, the resolution of the $H_{\infty}$ problem defined by the first inequality of (23) gives a filter $F$ such that condition (ii) of Corollary 1 is satisfied:

$$
F(s)=\frac{K(s)}{K_{p d}}=\frac{0.64(s+205.9)}{s}
$$

The global system stability is confirmed by the maximum real part of its poles: -75.9. Fig.8 reveals that with this filter, neither the local system (blue dashed line) or the global system (blue full line) satisfies the performance specification (red dotted line).

We now focus on the design of a filter $F$ such that the global system performance specification is satisfied by applying Theorem 3 . Let us choose $\beta=r^{2} \cdot \eta_{0}^{2}=316$ with $\eta_{0} \approx 19.65$, that is $26 \mathrm{~dB}$ which is an upper bound on the difference between $\left|S_{g l o}(j \omega)\right|$ and $\left|S_{l o c}(j \omega)\right|$ for the previously computed filter $F$, see Fig.8. By solving the optimization problem (20), 


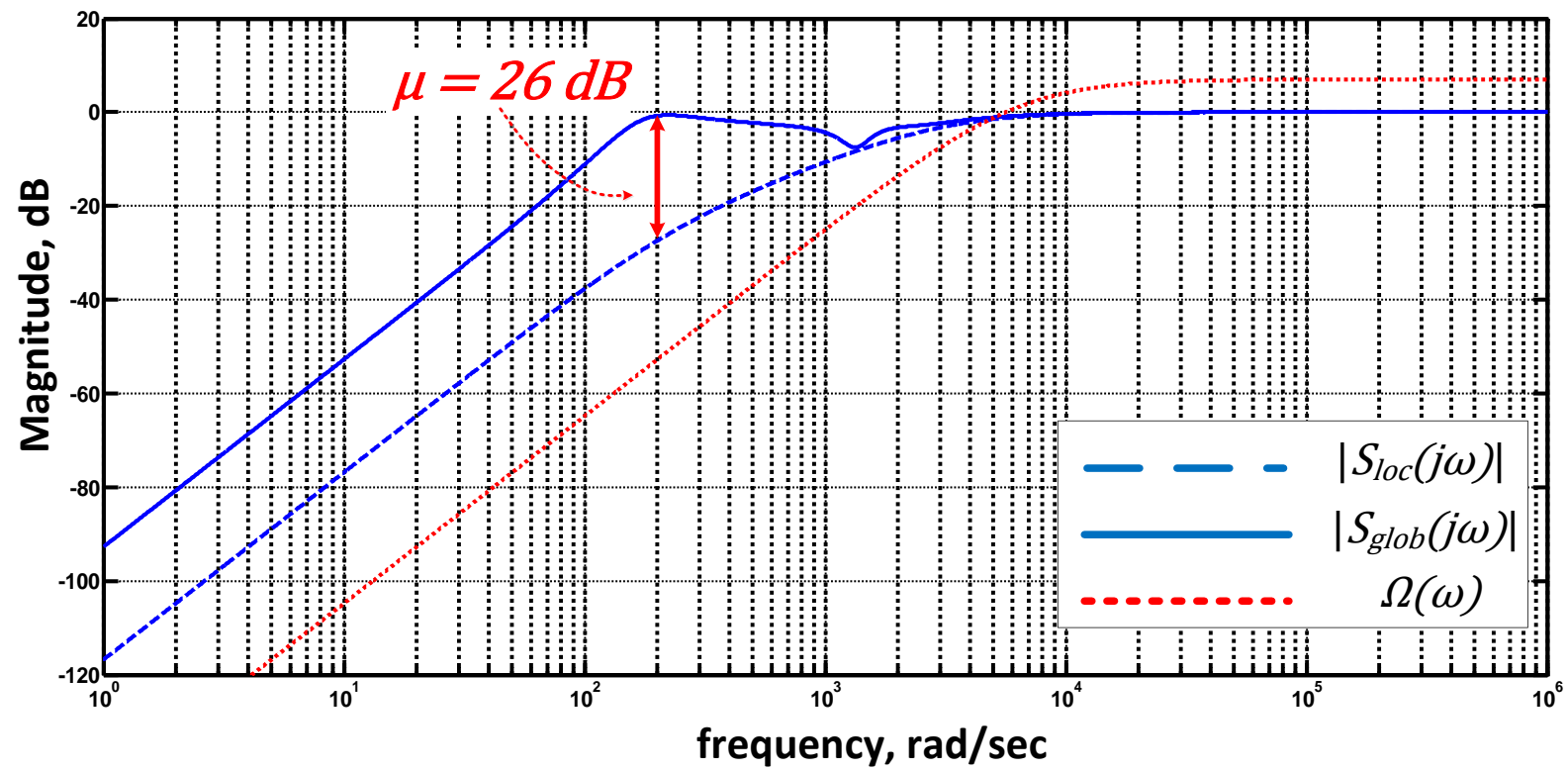

Figure 8: The magnitude of the local performance transfer function $S_{l o c}$ (blue dashed line), of the global performance transfer function $S_{\text {glob }}$ (blue full line) and of the corresponding global performance frequency constraint (red dotted line) 


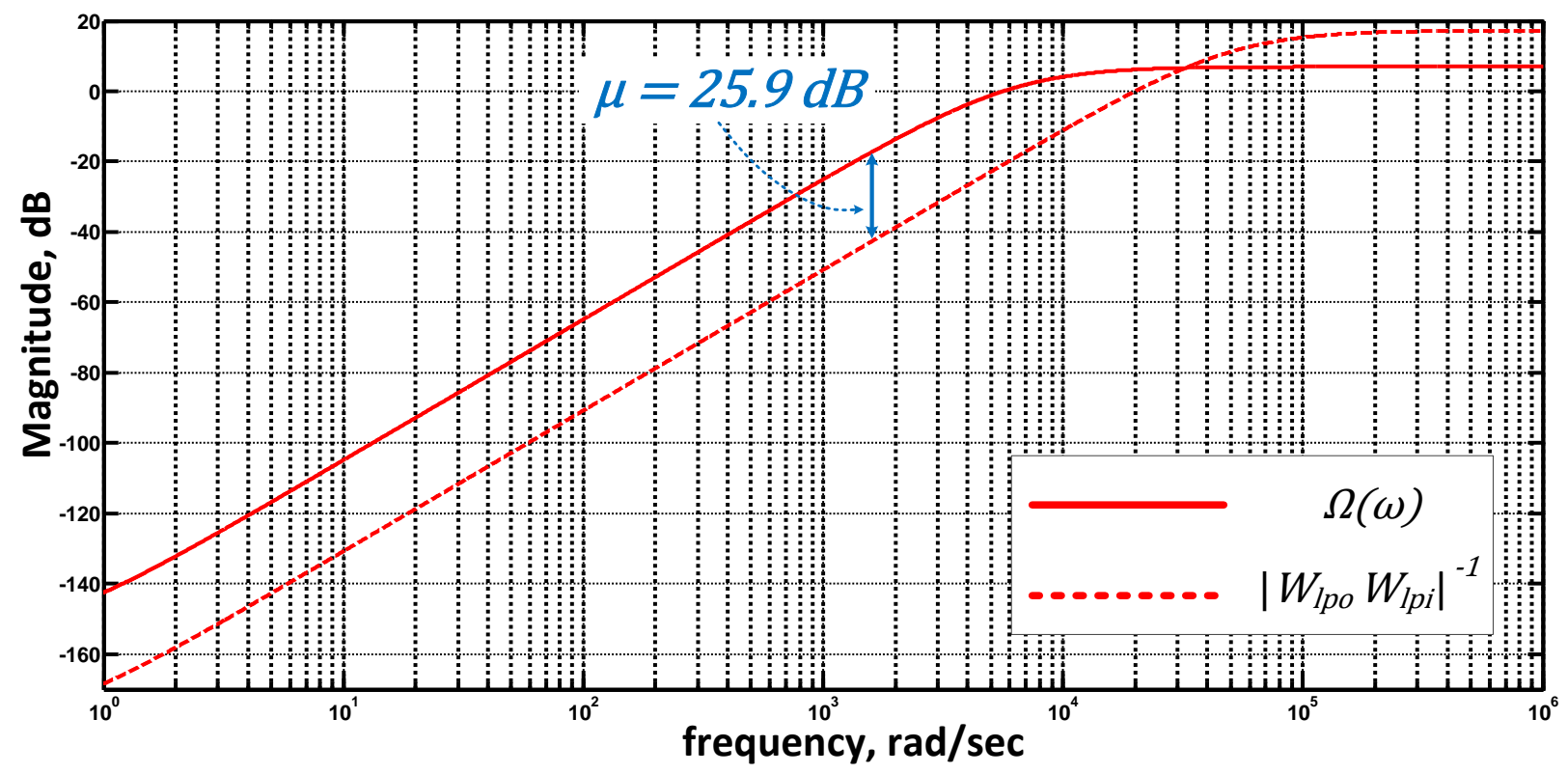

Figure 9: Chosen local (red dashed line) and global (red full line) frequency constraints we obtain: $r=0.905, X=-1.236, Y=0.124, Z=1, \eta \approx 19.63$, and that is $25.9 \mathrm{~dB}$.

The weighting functions $W_{l p i}, W_{l p o}$ are chosen such that (32) is satisfied:

$$
\left(W_{l p o}(s) W_{l p i}(s)\right)^{-1}=\frac{7.28(s+0.56)^{2}}{(s+50310)^{2}}
$$

see Fig.9. Note that the difference between two constraints in logarithmic scale is equal to $25.9 \mathrm{~dB}$ for the whole frequency range except the high frequencies. Nevertheless, since in the high frequency range, the magnitude of the global transfer function $S_{\text {glob }}$ is closed to 0 $\mathrm{dB}$, see [50], this issue is not critical. The additional local weighting functions $W_{1(2)}^{o}$ and $W_{1(2)}^{i}$ are chosen as:

$$
\begin{array}{ll}
W_{1}^{i}(s)=\left(9.22 \cdot 10^{-7}\right. & W_{2}^{i}(s)=6.34 \cdot 10^{-6} ; \\
W_{1}^{o}(s)=\frac{2 \cdot 10^{5}\left(s+1.23 \cdot 10^{6}\right)}{s+273.9} & W_{2}^{o}(s)=1010 .
\end{array}
$$

Since $S_{l o c}=G_{l p} \star K$ is the first input, first output transfer function of $\widehat{G} \star K$, a first choice of 
$W_{1}^{o}$ and $W_{1}^{i}$ can be such that $\left|W_{1}^{o} W_{1}^{i}\right| \approx\left|W_{l p o} W_{l p i}\right|$. In this case, from equation (34), $W_{1}^{o} W_{1}^{i}$ has to be a transfer function of order at least 2 , which is not the case. The main reason is that it is interesting to limit the order of the weighting functions, in order to obtain a filter of the smallest order. For this purpose, note that, from (33), $\left\|W_{1}^{o} \operatorname{Pr} T_{l p} W_{2}^{i}\right\|_{\infty}<1$. Then, $\left\|W_{l p o} G_{l p} \star K W_{l p i}\right\|_{\infty}<1$ can be ensured by choosing $W_{1}^{o}$ and $W_{2}^{i}$ such that $W_{1}^{o} \operatorname{Pr} W_{2}^{i} \approx$ $W_{l p o} W_{l p i}$. As $\operatorname{Pr}(s)=\frac{K_{V C O} / d}{s}, W_{1}^{i}, W_{2}^{i}$ and $W_{2}^{o}$ can be chosen constant and $W_{1}^{o}$ a transfer function of order 1 .

The resolution of the standard $H_{\infty}$ problem (29) leads to the filter:

$$
F(s)=\frac{49.7(s+1483)}{s}
$$

The global stability, the local performance specification (26), the additional local performance specification (33) and the global performance (24) are ensured with this filter. The local performance transfer functions with the corresponding constraints are represented in Fig.10.

The evolution of the magnitude of global performance transfer function $S_{g l o b}$ (dashed blue lines) along the different iterations is illustrated in the Fig.11. The cut-off frequency and Low Frequency (LF) range gain change until the global performance specification is reached. The result of the last iteration is represented by the full red line.

The results of the last iteration are presented in details in the Fig.12. The magnitude of the local performance transfer function $S_{l o c}$ (blue dashed line) satisfies the local frequency constraint $\left|W_{l p o}(j \omega) W_{l p i}(j \omega)\right|^{-1}$ (red dashed line). Furthermore the magnitude of the global performance transfer function $S_{g l o b}$ (blue full line) satisfies the local frequency constraint 

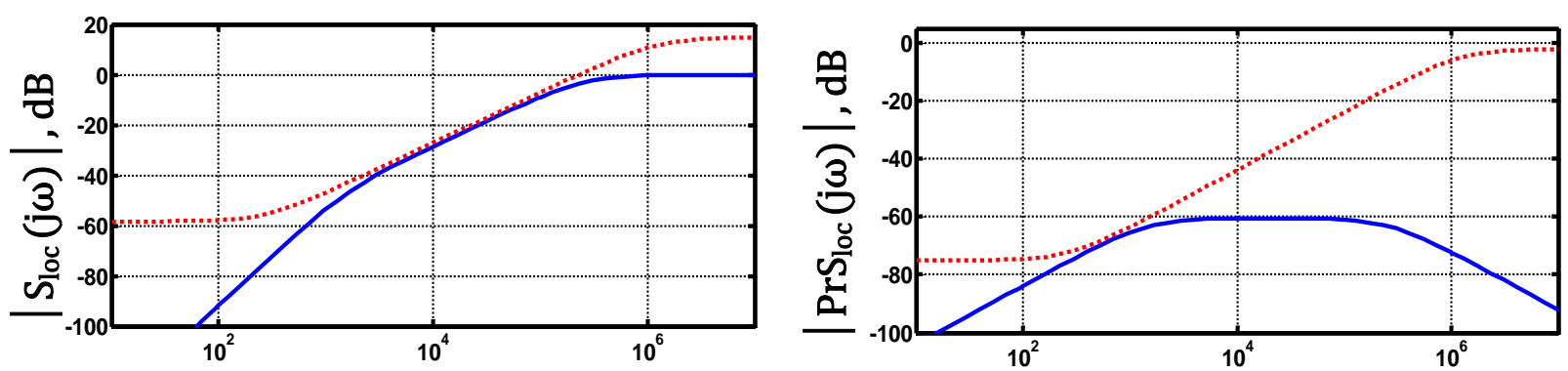

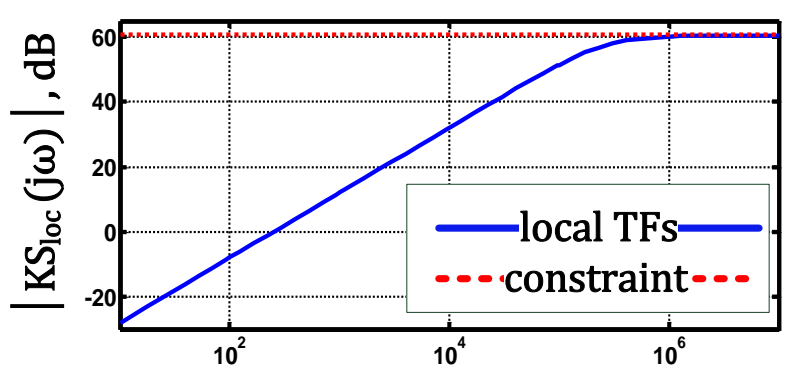

$\omega, \mathrm{rad} / \mathrm{sec}$

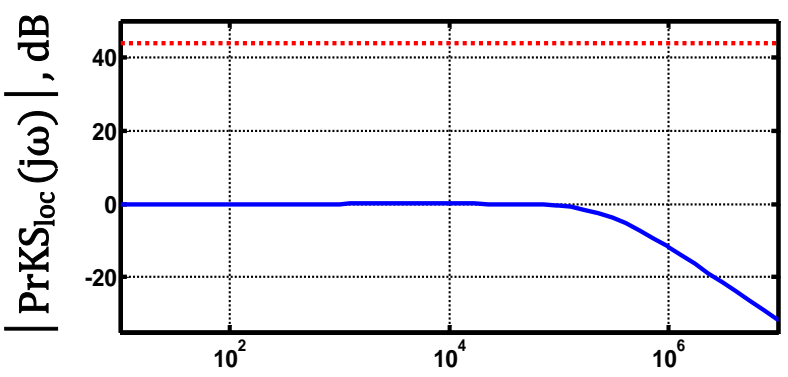

$\omega, \mathrm{rad} / \mathrm{sec}$

Figure 10: Additional local performance transfer function magnitudes (full blue lines) and corresponding constraints $\left|W_{1(2)}^{o}(j \omega) W_{1(2)}^{i}(j \omega)\right|^{-1}$ (dotted red lines) 


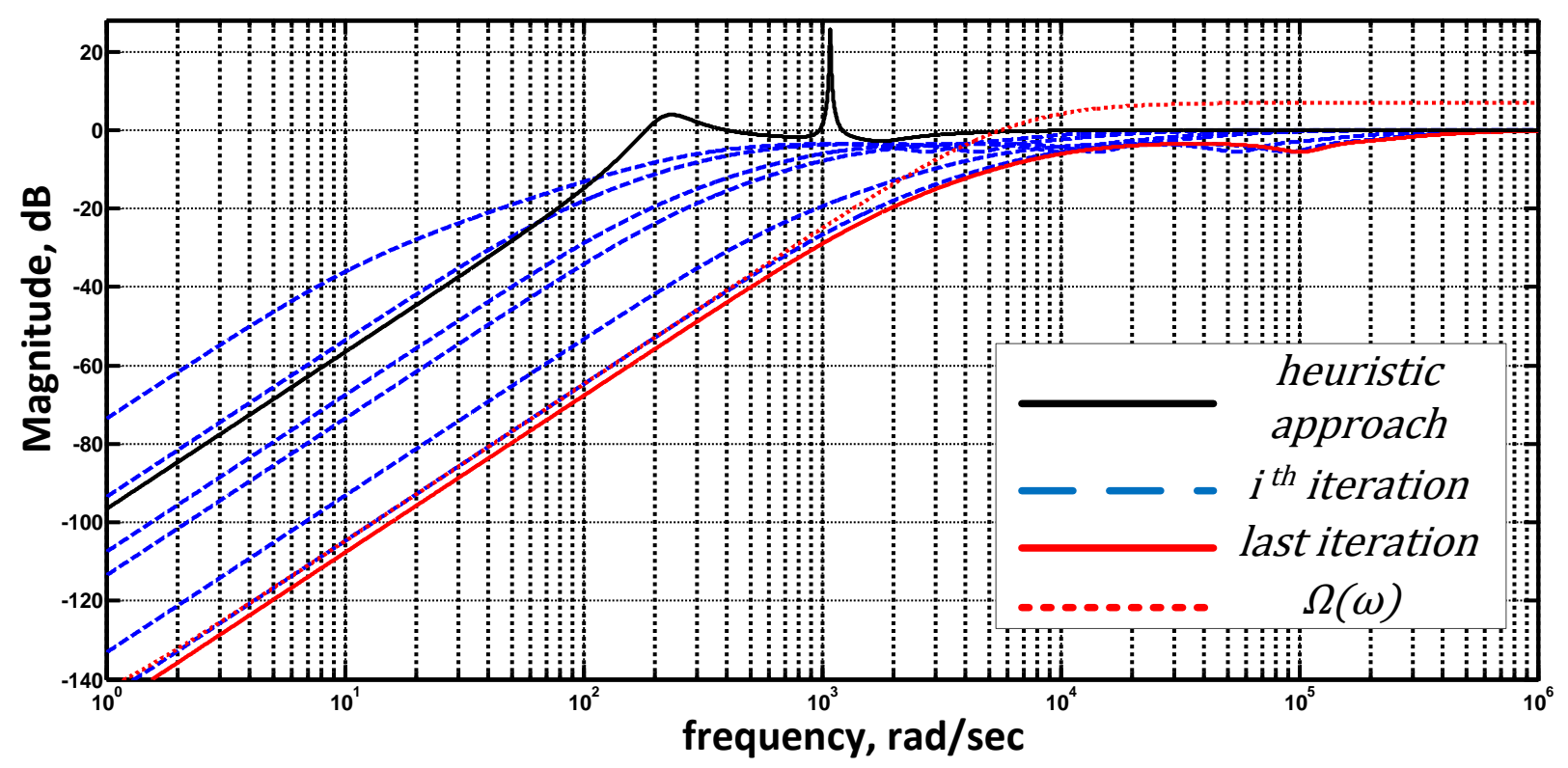

Figure 11: Obtained global performance transfer function magnitudes as a result of the heuristic design approach (black full line), of intermediate iteration (blue dashed lines) and last iteration (red full line) of the proposed design approach, the global frequency constraint (red dotted line)

$\left|S_{l o c}(j \omega)\right|_{d B}+20 \log (\eta)$ (green dashed-dotted line). Since the constraint $\left|S_{l o c}(j \omega)\right|+20 \log (\eta)$ is lower than the global performance frequency constraint $\Omega(j \omega)$ (full red line), the global performance specification is satisfied.

The benefit of the proposed design process with respect to the traditional one is illustrated by Fig. 13 where the magnitudes of the frequency response of global (full lines) and local (dashed lines) transfer functions are represented for the traditional process (black lines) and the proposed process (blue lines). In Fig.14 is represented the tracking error in the time domain with both approaches. We observe that the time response and the damping are dramatically improved. 


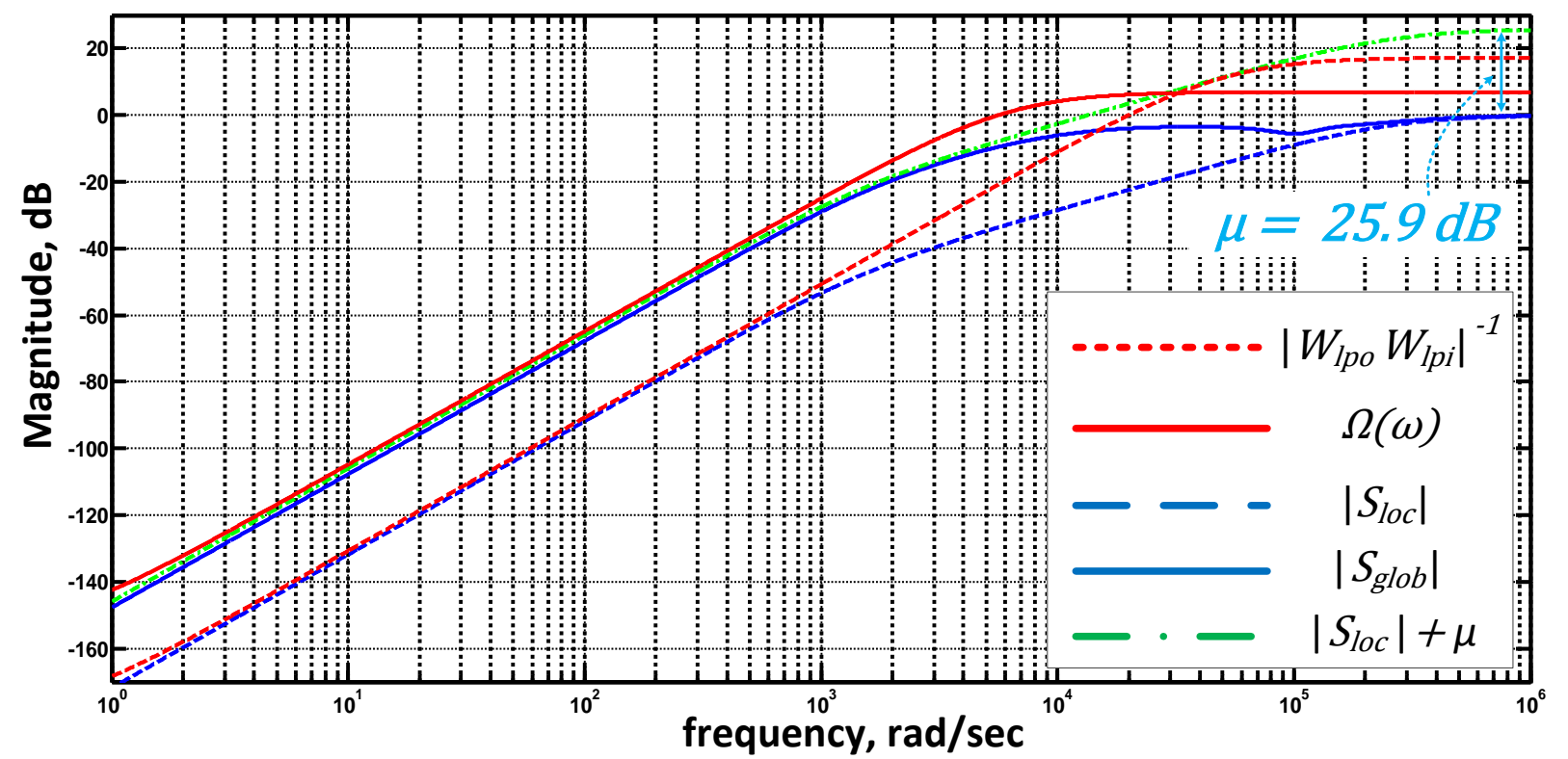

Figure 12: Magnitude of the local performance transfer function $S_{l o c}$ (blue dashed line) and of the global performance transfer function $S_{\text {glob }}$ (blue full line), local frequency constraint $\left|W_{l p o}(j \omega) W_{l p i}(j \omega)\right|^{-1}$ (red dashed line), frequency constraint imposed by $\left|S_{l o c}\right|+\mu$ (green dashed-dotted line) and the global performance frequency constraint (full red line) $\Omega(\omega)$ 


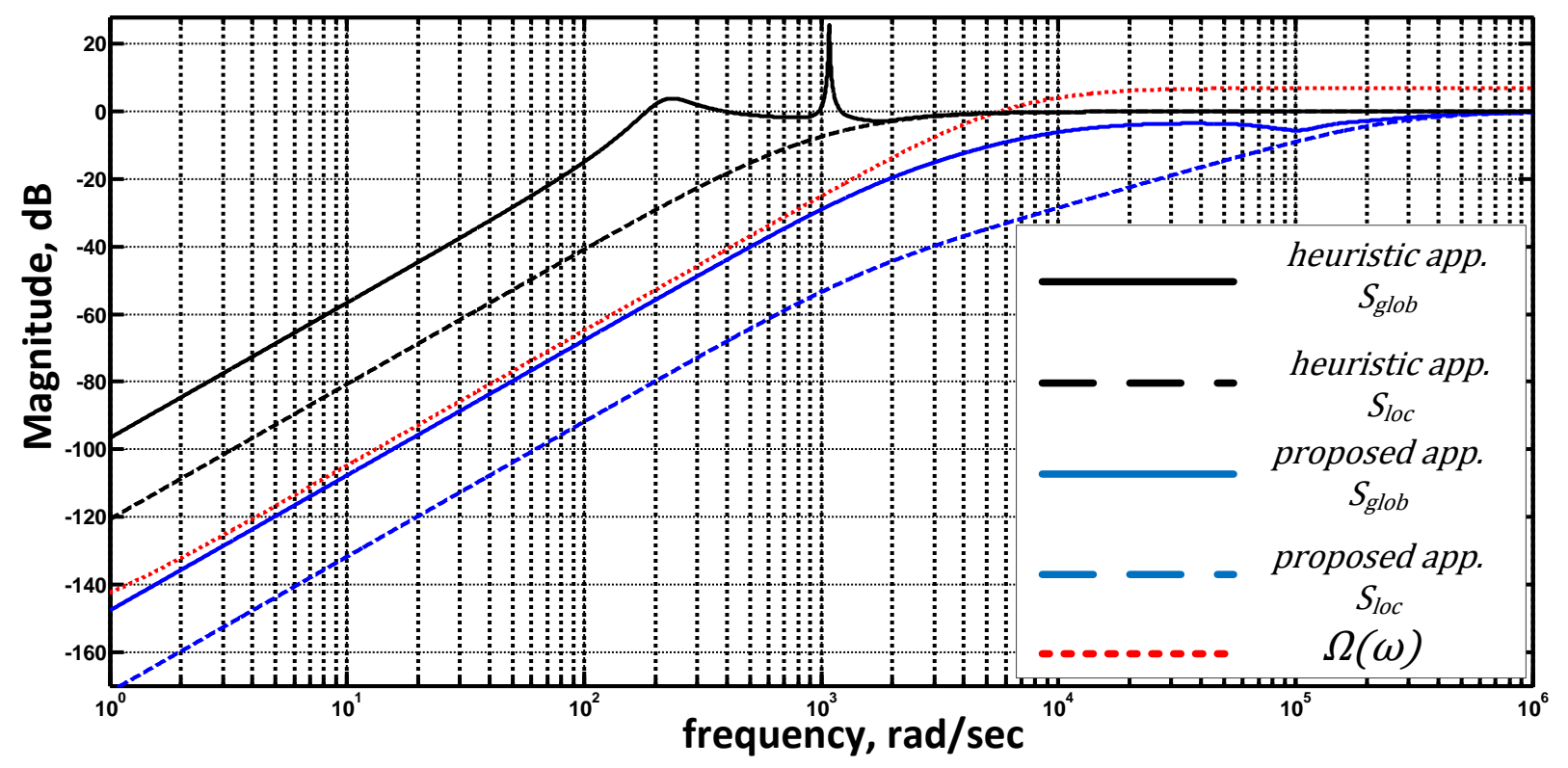

Figure 13: Magnitudes of designed global (full lines) and local (dashed lines) transfer functions for both heuristic (black lines) and proposed relative performance design (blue lines) approaches with corresponding global performance frequency constraint $\Omega(\omega)$ (dotted red line). 


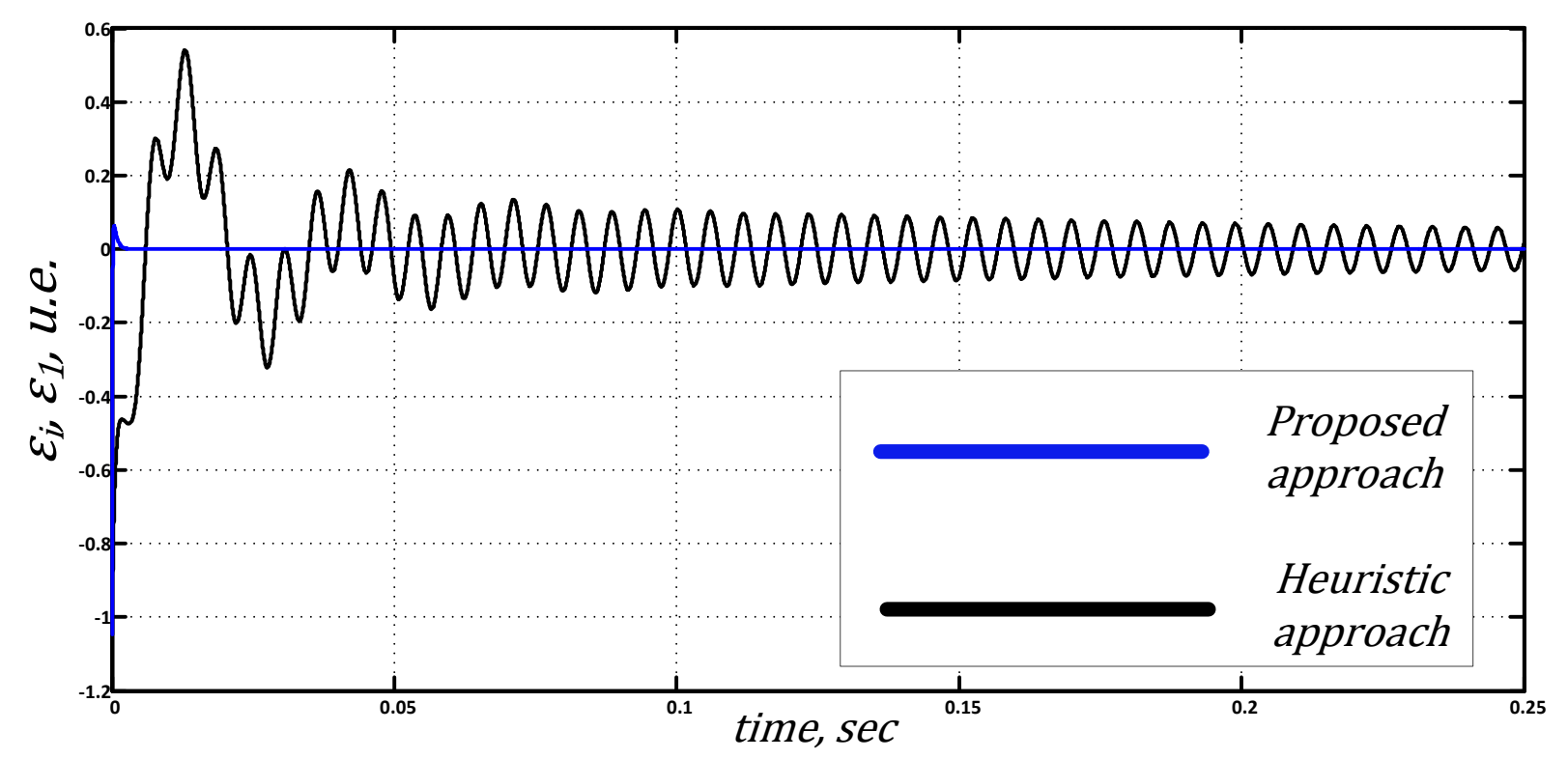

Figure 14: Temporal simulations results for both heuristic (black line) and proposed (blue line) design approaches

\section{Conclusion}

A new control law design method for large scale LTI systems defined as the given interconnection of identical subsystems is presented in this paper. The design method enforces a general global performance specification by designing a local controller such that the (stand-alone) closed loop subsystem satisfies performance specifications. This approach is motivated by improving the traditional design process of PLL network, rather than proposing a totally different one in order to ensure the acceptability of the method by the designers. The application to the design of a PLL network reveals that in addition to a priori ensure global stability and performance, a high level of performance is achieved with respect to the traditional design. The proposed design procedure has some common features with the 
traditional iterative, "trial and error" approaches to decentralized control [6]. Thanks to the a priori guarantees of global stability and performance, it is a nice alternative to these traditional approaches.

\section{Acknowledgement}

This work was supported by the French Agence Nationale de la Recherche (ANR).

\section{References}

[1] Cao Y, Yu W, Ren W, Chen G. An overview of recent progress in the study of distributed Multi-Agent coordination. Industrial Informatics, IEEE Transactions on Feb 2013; 9(1):427-438, doi:10.1109/TII.2012.2219061.

[2] Pratt GA, Nguyen J. Distributed synchronous clocking. IEEE Trans. Parallel Distrib. Syst. March 1995; 6(3):314-328.

[3] Gutnik V, Chandrakasan A. Active GHz clock network using distributed PLLs. IEEE J. Solid-State Circuits Nov 2000; 35(11):1553 -1560.

[4] Saint-Laurent M, Swaminathan M. A multi-PLL clock distribution architecture for gigascale integration. Proc. IEEE Computer Society Workshop on VLSI, WVLSI '01, IEEE Computer Society: Washington, 2001; 30-35. 
[5] Korniienko A, Colinet E, Scorletti G, Blanco E, Galayko D, Juillard J. A clock network of distributed ADPLLs using an asymmetric comparison strategy. Proc. Int. Symposium Circuits and Systems, Paris, 2010; 3212-3215.

[6] Lasley E, Michel A. Input-output stability of interconnected systems. IEEE Trans. Aut. Control february 1976; :84-89.

[7] Blondel V, Tsitsiklis JN. NP-hardness of some linear control design problems. SIAM J. of Control and Opt. 1997; 35(6).

[8] Fax J, Murray R. Information flow and cooperative control of vehicle formations. IEEE Trans. Aut. Control Sep 2004; 49(9):1465 - 1476.

[9] Wieland P, Kim J, Scheu H, Allgöwer F. On consensus in multi-agent systems with linear high-order agents. Proceedings of the 17th IFAC World Congress, Seoul, 2008; $1541-1546$.

[10] Wieland P, Allgöwer F. On consensus among identical linear systems using inputdecoupled functional observers. Proc. American Control Conf., Baltimore, 2010; 1641 $-1646$.

[11] Wieland P. From static to dynamic couplings in consensus and synchronization among identical and non-identical systems. PhD Thesis, Institut für Systemtheorie und Regelungstechnik Universität Stuttgart Sep 2010.

[12] Scardovi L, Sepulchre R. Synchronization in networks of identical linear systems. Automatica 2009; 45(11):2557 - 2562. 
[13] Wang J, Liu Z, Hu X. Consensus of high order linear multi-agent systems using output error feedback. Proc. IEEE Conf. on Decision and Control jointly with Chinese Control Conf., Shanghai, 2009; $3685-3690$.

[14] Blondel V, Gevers M. Simultaneous stabilizability of three linear systems is rationally undecidable. Mathematics of Control, Signals, and Systems (MCSS) 1993; 6:135-145.

[15] Zhai G, Okuno S, Imae J, Kobayashi T. A matrix inequality based design method for consensus problems in multi-agent systems. Int. J. Appl. Math. Comput. Sci. December $2009 ; 19: 639-646$.

[16] Zhai G, Okuno S, Imae J, Kobayashi T. Extended consensus algorithm for multi-agent systems [brief paper]. Control Theory Applications, IET october 2010; 4(10):2232 2238 .

[17] Liu Y, Jia Y, Du J, Yuan S. Dynamic output feedback control for consensus of multiagent systems: An $\mathrm{H}_{\infty}$ approach. Proc. American Control Conf., St. Louis, 2009; 4470 $-4475$.

[18] Massioni P, Verhaegen M. Distributed control for identical dynamically coupled systems: A decomposition approach. Automatic Control, IEEE Transactions on 2009; 54(1):124-135.

[19] Skogestad S, Postlethwaite I. Multivariable Feedback Control, Analysis and Design. John Wiley and Sons Chischester, 2005.

[20] Lunze J. Feedback Control of Large Scale Systems. Prentice-Hall, 1991. 
[21] Sandell NR, Varaiya P, Athans M, Safonov MG. Survey of decentralized control methods for large scale systems. IEEE Trans. Aut. Control 1978; 23(2):108-128.

[22] Geromel J, Bernussou J, Peres P. Decentralized control through parameter space optimization. Automatica 1994; 30(10):1565-1578.

[23] Hovd M, Skogestad S. Sequential design of decentralized controllers. Automatica 1994; 30(10):1601-1607.

[24] Shiau J, Chow J. Robust decentralized state feedback control design using an iterative linear matrix inequality algorithm. 13th Triennal World Congres, 1996; 203-208.

[25] Shiau J, Chow JH. Structurally constrained $\mathrm{H}_{\infty}$ suboptimal control design using an iterative linear matrix inequality algorithm based on a dual design formulation. Tamkang Journal of Science and Engineering 1998; 1(2):133-143.

[26] Scorletti G, Duc G. An LMI approach to decentralized $H_{\infty}$ control. Int. J. Control $2001 ; 74(3): 211-224$.

[27] Moylan PJ, Hill DJ. Stability criteria for large-scale systems. IEEE Trans. Aut. Control Apr 1978; AC-23(2):143-149.

[28] Safonov MG. Stability and Robustness of Multivariable Feedback Systems. MIT Press: Cambridge, 1980.

[29] Goh K, Safonov M. Robust analysis, sectors and quadratic functionals. IEEE Conf. Decision and Control, IEEE (ed.), New Orleans, Louisiana, 1995. 
[30] I L. Large scale heterogeneous networks, the davis-wielandt shell and graph separation. SIAM Journal on Control and Optimization 2012; 50(4):1753-1774.

[31] Olfati-Saber R, Murray RM. Consensus problems in networks of agents with switching topology and time-delays. Automatic Control, IEEE Transactions on 2004; 49(9):15201533.

[32] Murray R. Recent research in cooperative control of multi-vehicle systems. J. Dynamic Systems, Measurement and Control 2007; Vol.129, No. 5:571-583.

[33] Liu Y, Jia $\mathrm{Y} . \mathrm{H}_{\infty}$ consensus control of multi-agent systems with switching topology: a dynamic output feedback protocol. Int. J. Control 2010; 83(3):527-537.

[34] Bracewell R. The Fourrier Transform and its Applications. McGraw-Hill: New York, 1965.

[35] Rantzer A. On the Kalman-Yakubovich-Popov lemma. Systems and Control Letters Jan 1996; 27(5).

[36] Scherer C, Gahinet P, Chilali M. Multiobjective output-feedback control via LMI optimization. IEEE Trans. Aut. Control Jul 1997; 42(7):896-911.

[37] Boyd S, Ghaoui LE, Feron E, Balakrishnan V. Linear matrix inequalities in system and control theory, vol. 15. Studies in Appl. Math. SIAM, 1994.

[38] Chopra N, Spong M. Passivity-based control of multi-agent systems. Advances in Robot Control: From Everyday Physics to Human-Like Movements 2006; :107-134. 
[39] Arcak M. Passivity as a design tool for group coordination. IEEE Trans. Aut. Control Aug 2007; 52(8):1380-1390.

[40] Bai H, Arcak M, Wen JT. Adaptive design for reference velocity recovery in motion coordination. Syst. Control Letters Aug 2008; 57(8):602-610.

[41] Chopra N, Spong MW. Delay-independent stability for interconnected nonlinear systems with finite $l_{2}$ gain. Proc. IEEE Conf. on Decision and Control, New Orleans, 2007; $3847-3852$.

[42] Scorletti G, Ghaoui LE. Improved LMI conditions for gain scheduling and related problems. Int. J. Robust and Nonlinear Control Aug 1998; 8(10):845-877.

[43] Brewer J. Kronecker products and matrix calculus in system theory. IEEE Transactions on Circuits and Systems 1978; CAS-25(9):772-780.

[44] Boyd S, Barratt C. Linear Controller Design: Limits of Performance. Prentice-Hall, 1991.

[45] Scorletti G, Fromion V. Introduction la commande multivariable des systmes : mthodes de synthse frquentielle $H_{\infty}$. Polycopi Universit/ENSI Caen disponible sur http://www.greyc.ismra.fr/LAP/Gerard_S/ENSI_comrob.html, 2001.

[46] Razavi B. Monolithic phase-locked loops and clock recovery circuits: theory and design. IEEE Press, John Wiley and Sons, 1996. 
[47] Rohde U. Microwave and wireless synthesizers: theory and design. A Wiley Interscience publication, John Wiley \& Sons, 1997.

[48] Razavi B. RF Microelectronics. Prentice Hall PTR, 1998.

[49] Kroupa V. Phase lock loops and frequency synthesis. John Wiley \& Sons, 2003.

[50] Tonetti S, Murray R. Limits on the network sensitivity function for multi-agent systems on a graph. Technical Report, California Institute of Technology, Pasadena 2009.

[51] Kharrat C, Colinet E, Voda A. $\mathrm{H}_{\infty}$ loop shaping control for pll-based mechanical resonance tracking in nems resonant mass sensors. Proc. IEEE Sensors, Lecce, 2008; 11351138.

[52] Font S. Méthodologie pour prendre en compte la robustesse des systèmes asservis: optimisation $H_{\infty}$ et approche symbolique de la forme standard. PhD Thesis, Université Paris XI Orsay, France 1995.

[53] Korniienko A, Scorletti G, Colinet E, Blanco E. Control law design for distributed multi-agent systems. Technical Report, Laboratoire Ampère, Ecole Centrale de Lyon 2011. URL http://hal .archives-ouvertes.fr/hal-00630543/en/. 